\title{
Large-activation-energy theory for premixed combustion under the influence of enthalpy fluctuations
}

\author{
Xuesong $\mathrm{Wu}$ \\ Department of Mathematics, Imperial College London, SW7 2AZ, UK \\ Parviz Moin \\ Department of Mechanical Engineering, Stanford University, CA 94305, USA
}

January 31, 2010

\begin{abstract}
This paper presents a mathematical theory for premixed combustion under the influence of enthalpy fluctuations in the oncoming fresh mixture. Based on the assumptions of large activation energy and small Mach number, an analysis of the thermal, hydrodynamic and acoustic regions of a flame is performed to derive an interactive system that describes, on the first-principles basis, the intricate coupling between the flame and its spontaneously emitted acoustic waves. The system, in its general form, is strongly nonlinear and requires a numerical attack. In this paper, it is employed to analyze several fundamental physical processes in relatively simple cases in order to provide useful insights into the role of enthalpy fluctuations in combustion. Firstly, the linear response of the flame to two- or three-dimensional small-amplitude enthalpy fluctuations is considered, and they are found to generate hydrodynamic motion. Secondly, enthalpy fluctuations are shown to radiate sound waves through their interaction with the flame. Thirdly, enthalpy fluctuations and the sound waves emitted by them modify the flame stability, and the analysis shows that a moderate level of enthalpy fluctuation may cause a strong subharmonic parametric instability. Finally, in the small-heat-release limit, an extended Michelson-Sivashinsky equation is derived to describe the nonlinear evolution of the flame under the influence of both the imposed enthalpy fluctuations and the induced acoustic waves. Numerical solutions suggest that the flame evolves into a time-periodic state and acquires a curved profile, which primarily vibrates in the longitudinal direction, while its overall shape remains almost unaltered.
\end{abstract}

\section{Introduction}

\subsection{Premixed combustion and instability}

Premixed combustion is known to be susceptible to large-scale acoustic instability, which manifests itself as intense pressure fluctuations with predominant spectral peaks at the characteristic acoustic frequencies of the combustor (e.g. Sivasegaram \& Whitelaw 1987). Such an instability can occur in aero- and rocket engines as well as in land-based gas turbines (e.g. Harrje \& Reardon 1972, Yu, Trouve \& Daily 1991, Richards \& Janus 1997), and has a number of detrimental effects. For example, unstable combustion produces undesired oscillatory load, which may lead to structural fatigue. The strong pressure 
fluctuation may cause flame 'flash-back' and/or 'blow-off'. These problems hinder the development of lean-burn gas turbine engines to reduce emission of $\mathrm{NO}_{\mathrm{X}}$, because burning in the lean limit is particularly prone to the instability. In practical applications, the instability has to be suppressed by passive (Schadow \& Gutmark 1992), or active control (Candel 2002, Dowling \& Morgans 2005).

It is generally recognised that combustion instability is essentially a self-excited oscillation sustained by a two-way coupling between the flame and acoustic modes of the chamber (e.g. Poinsot et al. 1987, Langhorne 1988, Candel 2002). The unsteady heat release from the flame leads to amplification of acoustic pressure when the two are "in phase' according to Rayleigh's criterion. Acoustic fluctuations, on the other hand, may affect the flame through kinematic, dynamic and chemo-thermal mechanisms, including

(a) acoustic velocity advects the flame front;

(b) acoustic acceleration acts on the flame through the unsteady Rayleigh-Taylor (R-T) effect (Markstein 1953);

(c) acoustic pressure directly modifies the burning rate (e.g. Peters \& Ludford 1984, McIntosh 1991);

(d) sound waves modulate the feeding rate of the fuel, causing fluctuations in the equivalence ratio of the mixture (Lieuwen \& Zinn 1998).

As is indicated above, combustion instability involves intricate coupling of several processes (chemical reaction, heat transport, hydrodynamics and acoustics), which take place on distinct spatial scales. Chemical reaction and heat transport occur within thin sheets, the characteristic thicknesses of which are much smaller than the Kolmogorov scale, while acoustic wavelengthes are much greater than the typical length scale of the energetic fluid motion. Direct numerical simulations (DNS) of combustion instability based on the reactive Navier-Stokes (N-S) equations represent therefore a formidable task.

Theoretical modelling of combustion instability has mostly taken a semi-empirical approach, which seeks to establish relations between the flame motion and heat release in a phenomenological manner; see Lieuwen (2003) and Ducruix et al. (2003) for reviews. An alternative approach, which describes flame-acoustic interactions on the basis of first principles, may be pursued by using the large-activation-energy asymptotic (AEA) approximations. Based on the assumptions of large Zeldovich number $\beta \gg 1$ and small Mach number $M \ll 1$, AEA was developed to characterize flame-flow interactions (Clavin 1994, 2000; Matalon 2007). Depending on the ratio of the characteristic length scale $h^{*}$ of the flow motion to the flame thickness $d$, two distinguished regimes may arise. The 'corrugate flamelet' regime occurs if $h^{*} / d \gg O(1)$, for which the flow-flame system acquires an asymptotic structure consisting of three zones: an $O(d / \beta)$ reaction zone, an $O(d)$ preheat zone, and an $O\left(h^{*}\right)$ hydrodynamic zone (Matalon \& Matkowsky 1982, Pelce \& Clavin 1982). The 'thin-reaction-zone' regime resumes if $h^{*} / d \sim O(1)$, for which the preheat and hydrodynamic zones collapse so that a two-zoned structure emerges.

Using the AEA approach, several authors have investigated mechanism (c) referred to above. Harten, Kapila \& Matkowsky (1984) considered the interaction of a flame with an acoustic wave, whose time scale is comparable to the transit time of the flame, $O\left(d / U_{L}\right)$, where $U_{L}$ is the laminar flame speed. McIntosh $(1991,1993)$ analysed the burning rate response to acoustic waves in several distinguished higher-frequency regimes. Clavin, Pelce \& He (1990) studied the back effect of the burning rate change on the acoustic field, and found that the closed-loop interaction leads to an exponential growth of the sound. 
The dynamic impact of an acoustic field on a flame, i.e. mechanism (b), was investigated mathematically by Markstein \& Squire (1955) and Searby \& Rochwerger (1991). They formulated and solved the stability problem of a flame subjected to an externally prescribed acoustic pressure, and showed that as the acoustic acceleration exceeds a threshold, it induces a violent subharmonic parametric instability. Pelce \& Rochwerger (1992) analyzed the acoustic instability of a (slightly) curved stationary flame, and showed that the unsteady heat release due to the flame surface-area change drives exponential growth of acoustic modes. The back effect of the latter on the flame was however not considered.

The AEA theories for flame-acoustic interactions mentioned above were formulated either for one-way coupling or for two-way coupling in a special case. A general asymptotic theory was presented by Wu, Wang, Moin \& Peters (2003) (referred to as WWMP hereafter) to describe the acoustic-flow-flame coupling in the 'corrugated flamelet' regime. Using this general formulation, they provide a unified description of the flame-acoustic coupling mechanisms of Clavin et al. (1990) and Pelce \& Rochwerger (1992).

\subsection{Role of acoustic and vortical disturbances in combustion instability}

External disturbances play a crucial role in both the onset and control of combustion instability. In the idealized situation of a uniform oncoming flow, an arbitrary smallamplitude disturbance can be decomposed into acoustic, vortical and entropy modes. In the presence of a deficient reactant, the entity of entropy generalizes to enthalpy, which is a suitable linear combination of the temperature and reactant mass fraction. It is therefore natural to investigate the interaction of a flame with each of three modes separately.

Acoustic disturbances have been studied extensively because they are the key component in flame-acoustic coupling. Flame motions and heat release have been measured in order to extract transfer functions relating the response to externally imposed acoustic disturbances (e.g. Ducruix, Durox \& Candel 2000, Schuller et al. 2002). Theoretically, semi-empirical models based on the so-called G-equation have been extended to describe the flame wrinkling caused by perturbations (e.g. Schuller, Durox \& Candel 2003).

In contrast to acoustic disturbances, vortical disturbances have received little attention as far as their role in combustion instability is concerned. The only experimental study is that of Baillot, Durox \& Prud'homme (1992), who measured the flame response to small-amplitude vortical disturbances and characterized their relationship using a transfer function. Recently, Wu \& Law (2009) analysed the impact of vortical disturbances on flame-acoustic coupling by adapting the general theory of WWMP. Their analysis shows that weak vortical disturbances may initiate the subharmonic parametric resonance between the flame and the spontaneously generated sound wave, causing an initially silent planar flame to evolve into a noisy and highly wrinkled state.

\subsection{Role of enthalpy disturbances in combustion instability}

Enthalpy disturbances may consist of both temperature and fuel mass fraction (equivalence ratio) fluctuations. The latter may arise due either to 'unmixedness' of the reactant (Jimenez et al. 2002), or to combustion-generated acoustic waves interfering with the fuel mixing section (e.g. Mongia, Dibble \& Lovett 1998). Its relevance in combustion instability was first recognised by Lieuwen \& Zinn (1998), who proposed the distinct mechanism (d) mentioned earlier. An empirical mathematical model, based on the assumption that the equivalence ratio fluctuation induced by the pressure is proportional to the acoustic velocity, was proposed by Lieuwen et al. (2001). Recent measurements indicate that 
pressure oscillations correlate strongly with the equivalence ratio fluctuation at the inlet (Zimmer \& Tachibana 2007), and the latter was the primary contributor to the unsteady heat release that causes instability (Lee, Kim \& Santavicca 2000, Weigand et al. 2007).

Enthalpy disturbances are deliberately generated in active control systems using secondary fuel injection, which is the most practical and efficient strategy because of its flexible implementation and the sensitive response of flame to enthalpy. (e.g. Sivasegaram et al. 1995). In closed-loop control, modulating the fuel rate by a few percent may reduce the noise level by over 20dB (Jones, Lee \& Santavicca 1999, Tachibana et al. 2007).

Theoretical work on the interaction of a flame with enthalpy fluctuations is surprisingly limited. Mikolaitis (1984) and Daou, Matalon \& Linan (2000) studied propagations of flames parallel and perpendicular to the enthalpy gradient respectively using AEA. Both analyses adopted the thermal-diffusive model of constant density. Cho \& Lieuwen (2005) analyzed the linear response of a conical flame to equivalence ratio perturbation using the G-equation. In this approach, the fluctuating equivalence ratio influences the flame kinematics through its effect on the flame speed, which is accounted for by adapting an empirical relation. DNS were performed recently by Birbaud et al. (2008) for the case of an open inverted V-flame responding to a fuel mass fraction oscillation imposed at the inlet. The calculation shows that moderate perturbations may induce highly nonlinear response and considerable modification to the hydrodynamic motion. The role of enthalpy fluctuations has also been studied by DNS in the context of partially premixed combustion (e.g. Jimenez et al. 2002 and references therein). These simulations were mostly restricted to small domains in two dimensions, and their interest was primarily in the effect on the overall heat release and flame geometry rather than in acoustic instability.

\subsection{The scope of the present study}

Given that the empirical approach neglects crucial acoustic, hydrodynamic and thermaldiffusional processes, and DNS on the other hand are too costly to be performed routinely, in this paper we shall derive, from the N-S equations for reactive flows, an AEA theory for premixed combustion, where enthalpy fluctuations with intensity $h \sim O(1)$ are present in the oncoming mixture. The constant- density assumption made in previous AEA analyses of the effect of non-homogeneous enthalpy on flames, will be lifted in our general formulation in order to account for the impact of enthalpy fluctuations on the hydrodynamic motion. The asymptotically reduced theory then provides a mathematical framework for analyzing, on the basis of first principles, the role of enthalpy fluctuations on the flame dynamics and flame-acoustic coupling.

The problem is formulated in $\S 2$, and the relevant asymptotic scalings are specified for the flamelet regime. An asymptotic flow structure, consisting of four distinct interacting zones, then emerges. Relevant jump conditions across the preheat zone are derived. In $\S 3$, we consider the interplay between the acoustic and hydrodynamics fields to derive a closed system, which describes the enthalpy-acoustic-flame interaction. In $\S 4$, the linear response of a planar flame to weak three-dimensional fluctuations are calculated. In $\S 5$, we study the sound generation by one-dimensional enthalpy fluctuations. In $\S 6$, it is shown that an enthalpy fluctuation and the sound generated by it may lead to a parametric instability of the flame. In $\S 7$, we derive, in the limit of small heat release, an evolution equation of the generalised Michelson-Sivashinsky type to describe the nonlinear development of a flame under the combined influence of the enthalpy perturbation and its spontaneously emitted sound waves. A summary and concluding remarks will be given in $\S 8$. 


\section{Formulation}

\subsection{Governing equations and scalings}

Consider the combustion of a premixed combustible mixture in a duct of width $h^{*}$ and length $l^{*} \gg h^{*}$. The fresh mixture enters the duct at a constant mean velocity $U_{-}^{*}$, and has a mean density $\rho_{-\infty}$ and temperature $\Theta_{-\infty}$. Small temperature and/or mass fraction fluctuations, which may be characterized as enthalpy disturbances, are present on the oncoming flow. For simplicity, a one-step irreversible exothermic chemical reaction is assumed. The gaseous mixture, which is assumed to obey the state equation for a perfect gas, consists of a single deficient reactant and an abundant component so that the progressive variable of the reaction can be taken to be the mass fraction of the former, $Y$, while the physical properties are determined by the latter.

Due to steady heat release, the mean temperature (density) behind the flame increases (decreases) to $\Theta_{\infty}\left(\rho_{\infty}\right)$. A key non-dimensional parameter is the Zeldovich number,

$$
\beta=E\left(\Theta_{\infty}-\Theta_{-\infty}\right) / \mathcal{R} \Theta_{\infty}^{2},
$$

where $E$ is the dimensional activation energy and $\mathcal{R}$ the universal gas constant. The flame propagates into the fresh mixture at a mean speed $U_{L}$, and it has an intrinsic thickness $d=D_{t h}^{*} / U_{L}$, where $D_{t h}^{*}$ is the thermal diffusivity. For later reference, we define the ratio $\delta$ and and the Mach number $M$ as

$$
\delta=d / h^{*}, \quad M=U_{L} / a^{*},
$$

where $a^{*}=\left(\gamma p_{-\infty} / \rho_{-\infty}\right)^{\frac{1}{2}}$ is the speed of sound, with $\gamma$ denoting the ratio of specific heats. Other relevant parameters are: the Prandtl number Pr, Lewis number Le, and the normalised gravity force

$$
G=g h^{*} / U_{L}^{2}
$$

Let $(x, y, z)$ and $t$ be space and time variables normalised by $h^{*}$ and $h^{*} / U_{L}$ respectively. The velocity $\mathbf{u} \equiv(u, v, w)$, density $\rho$, and temperature $\theta$ are non-dimensionalised by $U_{L}$, $\rho_{-\infty}$ and $\Theta_{-\infty}$ respectively. The non-dimensional pressure $p$ is introduced by writing the dimensional pressure as $\left(p_{-\infty}+\rho_{-\infty} U_{L}^{2} p\right)$. The velocity, pressure, temperature and fuel mass fraction satisfy the non-dimensional N-S equations for reactive flows with the reaction rate $\Omega$ being described by the Arrhenius law,

$$
\Omega=\rho Y \exp \left\{\beta\left(\frac{1}{\Theta_{+}}-\frac{1}{\theta}\right)\right\},
$$

where $\Theta_{+}=1+q$ is the adiabatic flame temperature with $q$ being the heat release parameter.

As usual, the mathematical theory will be developed by using the AEA approach, which is based on the assumption that the Zeldovich number is large, i.e. $\beta \gg 1$. The chemical reaction then occurs in a thin region of width $O(d / \beta)$ centred at the flame front. Assume that the flame front is given by $x=f(y, z, t)$. It is convenient to introduce a coordinate system attached to the front (Matalon \& Matkowsky 1982),

$$
\xi=x-f(y, z, t), \quad \eta=y, \quad \zeta=z,
$$

and to split the velocity $\mathbf{u}$ as $\mathbf{u}=u \mathbf{i}+\mathbf{v}$, where $\mathbf{i}$ is the unit vector along the duct. It is convenient to work with a rescaled enthalpy $h$, defined via the relation

$$
\theta+q Y=1+q+\beta^{-1} h .
$$


The N-S equations can be written as

$$
\begin{gathered}
\frac{\partial \rho}{\partial t}+\frac{\partial m}{\partial \xi}+\nabla \cdot(\rho \mathbf{v})=0 \\
\rho \frac{\partial u}{\partial t}+m \frac{\partial u}{\partial \xi}+\rho \mathbf{v} \cdot \nabla u=-\frac{\partial p}{\partial \xi}+\delta \operatorname{Pr}\left\{\triangle u+\frac{1}{3} \frac{\partial}{\partial \xi}\left(\frac{\partial s}{\partial \xi}+\nabla \cdot \mathbf{v}\right)\right\}-\rho G \\
\rho \frac{\partial \mathbf{v}}{\partial t}+m \frac{\partial \mathbf{v}}{\partial \xi}+\rho \mathbf{v} \cdot \nabla \mathbf{v}=-\nabla p+\nabla f \frac{\partial p}{\partial \xi}+\delta \operatorname{Pr}\left\{\Delta \mathbf{v}+\frac{1}{3}\left(\nabla-\nabla f \frac{\partial}{\partial \xi}\right)\left(\frac{\partial s}{\partial \xi}+\nabla \cdot \mathbf{v}\right)\right\} \\
\rho \frac{\partial h}{\partial t}+m \frac{\partial h}{\partial \xi}+\rho \mathbf{v} \cdot \nabla h=\delta \triangle h+\delta l \triangle \theta+\beta(\gamma-1) M^{2}\left\{\frac{\partial p}{\partial t}+m \frac{\partial p}{\partial \xi}+\rho \mathbf{v} \cdot \nabla p\right\} \\
\rho \frac{\partial \theta}{\partial t}+m \frac{\partial \theta}{\partial \xi}+\rho \mathbf{v} \cdot \nabla \theta=\delta \triangle \theta+\delta q \Omega+(\gamma-1) M^{2}\left\{\frac{\partial p}{\partial t}+m \frac{\partial p}{\partial \xi}+\rho \mathbf{v} \cdot \nabla p\right\} \\
1+\gamma M^{2} p=\rho \theta
\end{gathered}
$$

where $m=\rho s$,

$$
\begin{gathered}
s=u-f_{t}-\mathbf{v} \cdot \nabla f \\
\triangle=\left(1+|\nabla f|^{2}\right) \frac{\partial^{2}}{\partial \xi^{2}}+\nabla^{2}-\nabla^{2} f \frac{\partial}{\partial \xi}-2 \frac{\partial}{\partial \xi}(\nabla f \cdot \nabla)
\end{gathered}
$$

here the operators $\nabla$ and $\nabla^{2}$ are defined with respect to the transverse variables $\eta$ and $\zeta$.

The AEA approach requires the Lewis number Le to be close to unity, or more precisely

$$
\text { Le }=1+\beta^{-1} l \quad \text { with } \quad l=O(1) .
$$

As in Matalon \& Matkowsky (1982) and WWMP, the hydrodynamic motion is assumed to occur on the length scale $h^{*}$. The corrugated flamelet regime then corresponds to

$$
\delta=d / h^{*} \ll 1, \quad M \ll 1 .
$$

We assume that enthalpy fluctuation has intensity $h \sim O(1)$, which corresponds to an $O\left(\beta^{-1}\right)$ variation in temperature and/or fuel mass fraction. They produce an $O(1)$ effect on the flame and flow, but the asymptotic structure, consisting of four distinct yet interactive regions as shown in figure 1 of WWMP, remains valid. In addition to the thin $O(d / \beta)$ reaction and $O(d)$ preheat zones, there are also hydrodynamic and acoustic regions, which scale on $h^{*}$ and $\lambda^{*}=O\left(h^{*} / M\right)$ respectively.

The mathematical problem for enthalpy disturbances interacting with a flame turns out to be more complex than those for vortical and acoustic disturbances. The latter affect, to leading order, only the hydrodynamic region so that theories describing their impact on the flame and flame-acoustic coupling (e.g. WWMP and Wu \& Law 2009) can be developed by using the results of Matalon \& Matkowsky (1982). In contrast, enthalpy fluctuations penetrate into the preheat zone to reach the reaction sheet, thereby disturbing the chemical reaction and heat release. This implies that the results of Matalon \& Matkowsky (1982), which were obtained assuming enthalpy fluctuations are absent, are not applicable, and the preheat zone has to be analyzed in detail in order to derive relevant jump conditions to be imposed on the flow motion in the outer hydrodynamic region. 


\subsection{Preheat zone}

As is implied above, the variable describing the preheat zone can be defined as

$$
\widehat{\xi}=\xi / \delta
$$

The solution in this region expands as

$$
\left.\begin{array}{rl}
(\theta, h, m) & =\left(\widehat{\theta}_{0}, \widehat{h}_{0}, m_{0}\right)+\delta\left(\widehat{\theta}_{1}, \widehat{h}_{1}, m_{1}\right)+\ldots, \\
(u, \mathbf{v}, p, f) & =\left(\widehat{u}_{0}, \widehat{\mathbf{v}}_{0}, \widehat{p}_{0}, f_{0}\right)+\delta\left(\widehat{u}_{1}, \widehat{\mathbf{v}}_{1}, \widehat{p}_{1}, f_{1}\right)+\ldots
\end{array}\right\}
$$

On the length scale of $d$, the reaction zone appears as a reaction sheet at $\widehat{\xi}=0$. Analysis of its internal structure on the length scale of $O(d / \beta)$ gives rise to the jump conditions (Matkowsky \& Sivashinsky 1979)

$$
\begin{array}{r}
{[h]=[\theta]=0, \quad[u]=[\mathbf{v}]=0, \quad\left[l \frac{\partial \theta}{\partial \widehat{\xi}}+\frac{\partial h}{\partial \widehat{\xi}}\right]=0,} \\
\left(1+|\nabla f|^{2}\right)^{1 / 2}\left[\frac{\partial \theta}{\partial \widehat{\xi}}\right]=-q \exp \left\{\frac{1}{2} h(0)\right\}, \\
{\left[\frac{\partial \mathbf{v}}{\partial \widehat{\xi}}+\frac{\partial u}{\partial \widehat{\xi}} \nabla f\right]=0, \quad[p]=\frac{4}{3} \operatorname{Pr}\left(1+|\nabla f|^{2}\right)\left[\frac{\partial u}{\partial \widehat{\xi}}\right],}
\end{array}
$$

where $[\cdot]$ denotes the jump of the flow quantity across the reaction zone.

The leading-order temperature and enthalpy, $\widehat{\theta}_{0}$ and $\widehat{h}_{0}$, are governed by the equations

$$
m_{0} \frac{\partial \widehat{\theta}_{0}}{\partial \widehat{\xi}}-\kappa \frac{\partial^{2} \widehat{\theta}_{0}}{\partial \widehat{\xi}^{2}}=0, \quad m_{0} \frac{\partial \widehat{h}_{0}}{\partial \widehat{\xi}}-\kappa \frac{\partial^{2} \widehat{h}_{0}}{\partial \widehat{\xi}^{2}}=l \kappa \frac{\partial^{2} \widehat{\theta}_{0}}{\partial \widehat{\xi}^{2}},
$$

where

$$
\kappa(\eta, \zeta, t)=1+\left|\nabla f_{0}\right|^{2} .
$$

The solution is found to be (cf. Matalon \& Matkowsky 1982)

$$
\begin{gathered}
\widehat{\theta}_{0}= \begin{cases}1+q \exp \left\{\frac{m_{0}}{\kappa} \widehat{\xi}\right\} & \widehat{\xi}<0, \\
1+q & \widehat{\xi}>0,\end{cases} \\
\widehat{h}_{0}= \begin{cases}h_{-\infty}-\frac{q l m_{0}}{\kappa} \widehat{\xi} \exp \left\{\frac{m_{0}}{\kappa} \widehat{\xi}\right\} & \widehat{\xi}<0, \\
h_{-\infty} & \widehat{\xi}>0,\end{cases} \\
m_{0}=\left(1+\left|\nabla f_{0}\right|^{2}\right)^{\frac{1}{2}} \exp \left\{\frac{1}{2} h_{-\infty}\right\},
\end{gathered}
$$

where $h_{-\infty}(\eta, \zeta, t)$ is a function to be determined by matching with the solution in the hydrodynamic zone. It represents the fluctuating enthalpy approaching the flame. In the work of Pelce \& Clavin (1982) and Matalon \& Matkowsky (1982), $h_{-\infty}$ is taken to be zero on the assumption that enthalpy disturbances are absent in the oncoming mixture. In the present work, $h_{-\infty} \neq 0$ and so the relation $\kappa=m_{0}^{2}$ does not holds. This is the main difference from that of Matalon \& Matkowsky (1982), but the ensuing analysis turns out to be similar to theirs. 
Consider now the velocity, which satisfies

$$
\begin{aligned}
m_{0} \frac{\partial \widehat{u}_{0}}{\partial \widehat{\xi}}-\operatorname{Pr}\left\{\kappa \frac{\partial^{2} \widehat{u}_{0}}{\partial \widehat{\xi}^{2}}+\frac{1}{3} \frac{\partial^{2} \widehat{s}_{0}}{\partial \widehat{\xi}^{2}}\right\} & =-\frac{\partial \widehat{p}_{0}}{\partial \widehat{\xi}} \\
m_{0} \frac{\partial \widehat{\mathbf{v}}_{0}}{\partial \widehat{\xi}}-\operatorname{Pr}\left\{\kappa \frac{\partial^{2} \widehat{\mathbf{v}}_{0}}{\partial \widehat{\xi}^{2}}-\frac{1}{3} \frac{\partial^{2} \widehat{s}_{0}}{\partial \widehat{\xi}^{2}} \nabla f_{0}\right\} & =-\nabla \widehat{p}_{0}+\frac{\partial \widehat{p}_{0}}{\partial \widehat{\xi}} \nabla f_{0} .
\end{aligned}
$$

The solution is found to be

$$
\begin{array}{r}
\left(\widehat{u}_{0}, \widehat{\mathbf{v}}_{0}\right)= \begin{cases}\left(u_{0}^{-}, \mathbf{v}_{0}^{-}\right)+\left(1,-\nabla f_{0}\right) \frac{q m_{0}}{\kappa} \exp \left\{\frac{m_{0}}{\kappa} \widehat{\xi}\right\} & \widehat{\xi}<0, \\
\left(u_{0}^{-}, \mathbf{v}_{0}^{-}\right)+\left(1,-\nabla f_{0}\right) \frac{q m_{0}}{\kappa} & \widehat{\xi}>0,\end{cases} \\
\widehat{p}_{0}= \begin{cases}p_{0}^{-}+\left(\frac{4}{3} \operatorname{Pr}-1\right) \frac{q m_{0}^{2}}{\kappa} \exp \left\{\frac{m_{0}}{\kappa} \widehat{\xi}\right\} & \widehat{\xi}<0, \\
p_{0}^{-}-\frac{q m_{0}^{2}}{\kappa} & \widehat{\xi}>0,\end{cases}
\end{array}
$$

where the integration constants $u_{0}^{-}, \mathbf{v}_{0}^{-}$and $p_{0}^{-}$, represent the velocity and pressure of the flow at the unburned side of the flame. Let $[[\cdot]]$ denote the difference of the indicated quantity across the preheat (flame) zone. Matching with the solution in the outer hydrodynamic region determines the leading-order jumps

$$
\left[\left[u_{0}\right]\right]=\frac{q m_{0}}{\kappa}, \quad\left[\left[\mathbf{v}_{0}\right]\right]=-\frac{q m}{\kappa} \nabla f_{0}, \quad\left[\left[p_{0}\right]\right]=-q \exp \left\{h_{-\infty}\right\}, \quad\left[\left[h_{0}\right]\right]=0 .
$$

Taking the limit $\widehat{\xi} \rightarrow-\infty$ in $(2.9)$ yields the leading-order front equation

$$
\frac{\partial f_{0}}{\partial t}=u_{0}^{-}-\mathbf{v}_{0}^{-} \cdot \nabla f_{0}-\left(1+\left|\nabla f_{0}\right|^{2}\right)^{\frac{1}{2}} \exp \left\{\frac{1}{2} h_{-\infty}\right\} .
$$

The analysis can be carried to next order to obtain the second-order jumps and the equation satisfied by $f_{1}$. The rather length derivation can be found in Wu \& Moin (2008). Here we only give the final results

$$
\begin{aligned}
& {\left[\left[\mathbf{v}_{1}+u_{1} \nabla f_{0}\right]\right]=}-\frac{q m_{0}}{\kappa} \nabla f_{1}+\operatorname{Pr} \frac{\kappa}{m_{0}}\left[\left[\frac{\partial}{\partial \xi}\left(\mathbf{v}_{0}+u_{0} \nabla f_{0}\right)\right]\right]+\frac{\kappa}{m_{0}^{2}} \ln (1+q) \mathbf{a}_{\mathrm{T}} \\
&-\frac{q}{\kappa}\left(\nabla f_{0} \cdot \nabla\right) \nabla f_{0}+\operatorname{Pr} \frac{q}{\kappa} \nabla \kappa-(\operatorname{Pr}-1) \frac{q}{m_{0}} \nabla m_{0}, \\
& {\left[\left[u_{1}\right]\right]=}-\frac{l q \mathcal{D}}{2 \kappa} \Gamma+\chi\left\{\frac{\kappa}{m_{0}}\left[\left[\frac{\partial u_{0}}{\partial \xi}\right]\right]-\frac{q}{\kappa} \nabla^{2} f_{0}+\frac{2 q m_{0}}{\kappa^{2}} \nabla f_{0} \cdot \nabla\left(\frac{\kappa}{m_{0}}\right)\right\} \\
&-\frac{q m_{0}}{\kappa^{2}} \nabla f_{0} \cdot \nabla f_{1}+\frac{q}{2 \kappa}\left(1-\frac{1+q}{q} \ln (1+q)\right) \nabla f_{0} \cdot \nabla h_{-\infty} \\
&+\frac{q}{2 \kappa}\left\{\frac{\kappa}{m_{0}}\left(\frac{q}{1+q}+\ln (1+q)\right) \frac{\tilde{D}}{\tilde{D} t} h_{-\infty}+m_{0} h_{1}^{-}\right\} \\
& {\left[\left[p_{1}\right]\right]=-2 m_{0}\left[\left[u_{1}\right]\right]+(\operatorname{Pr}+\chi)\left\{\kappa\left[\left[\frac{\partial u_{0}}{\partial \xi}\right]\right]-\frac{q m_{0}}{\kappa} \nabla^{2} f_{0}+\frac{2 q m_{0}^{2}}{\kappa^{2}} \nabla f_{0} \cdot \nabla\left(\frac{\kappa}{m_{0}}\right)\right\} }
\end{aligned}
$$




$$
\begin{aligned}
+ & \frac{q m_{0}}{\kappa} \nabla^{2} f_{0}-\frac{q m_{0}^{2}}{\kappa^{2}}\left(2 \nabla f_{0} \cdot \nabla f_{1}+\nabla f_{0} \cdot \nabla\left(\frac{\kappa}{m_{0}}\right)\right) \\
+ & \left\{\frac{\kappa}{m_{0}} \frac{\tilde{D} u_{0}^{-}}{\tilde{D} t}+\frac{m_{0}}{\kappa} \frac{\tilde{D}}{\tilde{D} t}\left(\frac{\kappa}{m_{0}}\right)+G\left(\frac{\kappa}{m_{0}}\right)\right\} \ln (1+q) \\
+ & \frac{q m_{0}}{2 \kappa}\left(1-\frac{1+q}{q} \ln (1+q)\right) \nabla f_{0} \cdot \nabla h_{-\infty}, \\
{\left[\left[h_{1}\right]\right]=} & \left(\ln (1+q)+\frac{q}{1+q}\right) \frac{\kappa}{m_{0}^{2}} \frac{\tilde{D}}{\tilde{D} t} h_{-\infty}-\frac{l \mathcal{D}}{m_{0}} \Gamma, \\
\frac{\partial f_{1}}{\partial t}+\mathbf{v}_{0}^{-} \cdot \nabla f_{1}+\mathbf{v}_{1}^{-} \cdot \nabla f_{0}= & u_{1}^{-}-\frac{m_{0}}{\kappa} \nabla f_{0} \cdot \nabla f_{1}+\mathrm{M}_{a} \Gamma \\
& \quad-\frac{1}{2}\left\{\frac{\kappa}{m_{0}}\left(\ln (1+q)+\frac{q}{1+q}\right) \frac{\tilde{D}}{\tilde{D} t} h_{-\infty}+m_{0} h_{1}^{-}\right\},
\end{aligned}
$$

where we have put

$$
\begin{gathered}
\mathbf{a}_{\mathrm{T}}=\frac{\tilde{D} \mathbf{v}_{0}^{-}}{\tilde{D} t}+\nabla f_{0} \frac{\tilde{D} u_{0}^{-}}{\tilde{D} t}+\frac{m_{0}}{\kappa} \frac{\tilde{D}}{\tilde{D} t} \nabla f_{0}+G \nabla f_{0}, \quad \Gamma(\eta, \zeta, t)=\frac{D}{D t}\left(\frac{\kappa}{m_{0}}\right)+\frac{\kappa}{m_{0}} \nabla \cdot \mathbf{v}_{0}^{-}+\nabla^{2} f_{0}, \\
\frac{D}{D t}=\frac{\partial}{\partial t}+\mathbf{v}_{0}^{-} \cdot \nabla, \quad \frac{\tilde{D}}{\tilde{D} t}=\frac{D}{D t}+\frac{m_{0}}{\kappa}\left(\nabla f_{0} \cdot \nabla\right), \\
\mathcal{D}(q)=\int_{0}^{\infty} \ln \left(1+q \mathrm{e}^{-x}\right) \mathrm{d} x, \quad \chi=\operatorname{Pr}+\frac{1+q}{q} \ln (1+q), \quad \mathrm{M}_{a}=\frac{1+q}{q} \ln (1+q)+\frac{1}{2} l \mathcal{D} .
\end{gathered}
$$

Expressions for $\left[\left[\frac{\partial}{\partial \xi}\left(\mathbf{v}_{0}+u_{0} \nabla f_{0}\right)\right]\right]$ and $\left[\left[\frac{\partial u_{0}}{\partial \xi}\right]\right]$ will be derived in $\S 2.3$; see $(2.33)-(2.34)$.

\subsection{Hydrodynamic zone}

In the hydrodynamic zone, the mean density $R=R_{-}=1$ for $\xi<0$ and $R=R_{+}=1 /(1+q)$ for $\xi>0$. The solution for the flow field, the flame interface and the enthalpy, expands as

$$
(u, \mathbf{v}, p, f, h)=\left(u_{0}, \mathbf{v}_{0}, p_{0}, f_{0}, h_{0}\right)+\delta\left(u_{1}, \mathbf{v}_{1}, p_{1}, f_{1}, h_{1}\right)+\ldots
$$

The expansion should also consist of $O(\beta M)$ terms because the acoustic pressure contributes an $O(\beta M)$ correction to the flame speed (WWMP). In order to avoid complicating further an already complex analysis, this effect is neglected here, although it may be taken into account by tactically assuming $\beta M=O(\delta)$ (WWMP).

Substitution of (2.31) into (2.3)-(2.5) leads to the equations governing $\left(u_{0}, \mathbf{v}_{0}, p_{0}\right)$ :

$$
\left.\begin{array}{rl}
\frac{\partial s_{0}}{\partial \xi}+\nabla \cdot \mathbf{v}_{0} & =0, \\
R\left\{\frac{\partial u_{0}}{\partial t}+s_{0} \frac{\partial u_{0}}{\partial \xi}+\mathbf{v}_{0} \cdot \nabla u_{0}\right\} & =-\frac{\partial p_{0}}{\partial \xi}-R G, \\
R\left\{\frac{\partial \mathbf{v}_{0}}{\partial t}+s_{0} \frac{\partial \mathbf{v}_{0}}{\partial \xi}+\mathbf{v}_{0} \cdot \nabla \mathbf{v}_{0}\right\} & =-\nabla p_{0}+\nabla f_{0} \frac{\partial p_{0}}{\partial \xi},
\end{array}\right\}
$$

where $s_{0}=u_{0}-f_{0, t}-\mathbf{v}_{0} \cdot \nabla f_{0}$. The momentum equations for $u_{0}$ and $\mathbf{v}_{0}$ can be combined to eliminate the pressure $p_{0}$, leading to (Matalon \& Matkowsky 1982)

$$
m \frac{\partial}{\partial \xi}\left(\mathbf{v}_{0}+u_{0} \nabla f_{0}\right)+R \nabla f_{0}\left(\frac{\partial}{\partial t}+\mathbf{v}_{0} \cdot \nabla\right) u_{0}+R\left(\frac{\partial}{\partial t}+\mathbf{v}_{0} \cdot \nabla\right) \mathbf{v}_{0}=-\nabla p_{0}-R G \nabla f_{0}
$$


Taking the limits $\xi \rightarrow 0^{ \pm}$, and subtracting the results, we obtain

$$
\left[\left[\frac{\partial}{\partial \xi}\left(\mathbf{v}_{0}+u_{0} \nabla f_{0}\right)\right]\right]=\frac{q}{(1+q) m_{0}} \mathbf{a}_{\mathrm{T}}-\frac{q m_{0}}{\kappa^{2}}\left(\nabla f_{0} \cdot \nabla\right) \nabla f_{0}+\frac{q}{m_{0}} \nabla\left(\frac{m_{0}^{2}}{\kappa}\right) .
$$

The continuity equation implies that $\left[\left[\frac{\partial u_{0}}{\partial \xi}-\frac{\partial \mathbf{v}_{0}}{\partial \xi} \cdot \nabla f_{0}\right]\right]=-q \nabla \cdot\left(\frac{m_{0}}{\kappa} \nabla f_{0}\right)$, which may be re-written as

$$
\left[\left[\frac{\partial u_{0}}{\partial \xi}\right]\right]=\frac{\nabla f_{0}}{\kappa} \cdot\left[\left[\frac{\partial}{\partial \xi}\left(\mathbf{v}_{0}+u_{0} \nabla f_{0}\right)\right]\right]+\frac{q}{\kappa} \nabla \cdot\left(\frac{m_{0}}{\kappa} \nabla f_{0}\right) .
$$

Equations (2.32) are to be solved subject to the jump conditions (2.24), which involves $h_{-\infty}$. To determine $h_{-\infty}$, we need to solve the transport equation of the enthalpy

$$
\frac{\partial h_{0}}{\partial t}+s_{0} \frac{\partial h_{0}}{\partial \xi}+\mathbf{v}_{0} \cdot \nabla h_{0}=0
$$

It follows from matching requirement that $h_{-\infty}=h_{0}(0, \eta, \zeta, t)$.

At $O(\delta),\left(u_{1}, \mathbf{v}_{1}, p_{1}, h_{1}\right)$ satisfies a linearized version of $(2.32)$ but consisting also of viscous terms. These equations will not be considered any further, because it is more convenient to construct a composite approximation by retaining the $O(\delta)$ viscous terms at leading-order, and imposing the jump conditions with $O(\delta)$ accuracy. The latter follow from combining (2.24) with (2.26)-(2.28). Note that $-\left(q m_{0} / \kappa^{2}\right) \nabla f_{0} \cdot \nabla f_{1}+q m_{0} h_{1}^{-} /(2 \kappa)$ in (2.27) can be absorbed into $q m / \kappa$ on the understanding that $f$ and $h_{-\infty}$ now stand for the two-term approximations: $f \approx f_{0}+\delta f_{1}$ and $h_{-\infty} \approx h_{-\infty}+\delta h_{1}^{-}$. On using (2.33)-(2.34), the longitudinal velocity jump may be written as

$$
\begin{aligned}
{[[u]]=} & \frac{q m}{\kappa}+\delta\left\{-\frac{q l \mathcal{D}}{2 \kappa} \Gamma+\frac{q \chi}{(1+q) m^{2}} \mathbf{a}_{\mathrm{T}} \cdot \nabla f\right. \\
& \left.+\frac{q}{2 \kappa}\left[(\operatorname{Pr}+1) \nabla f \cdot \nabla h_{-\infty}+\frac{\kappa}{m}\left(\frac{q}{1+q}+\ln (1+q)\right) \frac{\tilde{D} h_{-\infty}}{\tilde{D} t}\right]\right\} .
\end{aligned}
$$

Similarly, the transverse velocity and pressure jumps are found to be

$$
\begin{gathered}
{[[\mathbf{v}]]=-[[u]] \nabla f+\delta\left\{\frac{q \chi \kappa}{(1+q) m^{2}} \mathbf{a}_{\mathrm{T}}+\frac{1}{2}(\operatorname{Pr}+1) q \nabla h_{-\infty}\right\},} \\
{[[p]]=-q \exp \left\{h_{-\infty}\right\}-2 m[[u]]+\delta\left\{\left[\frac{\kappa}{m} \frac{\tilde{D} u^{-}}{\tilde{D} t}+\frac{m}{\kappa} \frac{\tilde{D}}{\tilde{D} t}\left(\frac{\kappa}{m}\right)+G \frac{\kappa}{m}\right] \ln (1+q)+\frac{q m}{\kappa} \nabla^{2} f\right.} \\
\left.-\frac{q m}{2 \kappa^{2}} \nabla f \cdot \nabla \kappa+\frac{q(\operatorname{Pr}+\chi)}{(1+q) m} \mathbf{a}_{\mathrm{T}} \cdot \nabla f+\frac{q m}{\kappa}(\operatorname{Pr}+1) \nabla f \cdot \nabla h_{-\infty}\right\} .
\end{gathered}
$$

The equation governing the flame front is

$$
f_{t}+\mathbf{v}^{-} \cdot \nabla f=u^{-}-m+\delta\left\{\mathrm{M}_{a} \Gamma-\frac{\kappa}{2 m}\left[\ln (1+q)+\frac{q}{1+q}\right] \frac{\tilde{D} h_{-\infty}}{\tilde{D} t}\right\} .
$$

Results (2.36)-(2.39) generalize those of Matalon \& Matkowsky (1982) with the latter being recovered when $h_{-\infty}=0$. As (2.39) indicates, enthalpy fluctuations modulate the local propagation speed of the flame. This is a well-known effect, which can be accounted for approximately by a thermal-diffusive model (Mikolaitis 1984, Daou et al. 2000). The 
velocity and pressure jumps (2.36)-(2.38) show that in conjunction with the gas-expansion effect enthalpy fluctuations may also generate a hydrodynamic motion. Capturing this coupling in a theoretical framework is important in view of the findings of DNS that enthalpy fluctuations change hydrodynamics significantly (Birbaud et al. 2008) and that the impact of enthalpy on the heat release and flame wrinkling would be severely underpredicted if the gas-expansion induced hydrodynamic instability is excluded (GarridoLopez \& Sarkar 2005).

\section{Acoustic-flame-enthalpy interaction}

The results obtained in $\S 2.3$ allow us to show that an unsteady flame emits spontaneous sound waves, which act simultaneously on the flame. This two-way coupling is primarily facilitated by the hydrodynamic motion as in WWMP, but is now modified by enthalpy fluctuations. A composite acoustic-flame-enthalpy interaction theory of second-order accuracy can be derived by retaining the $O(\delta)$ viscous terms in the hydrodynamic equations, and using the jumps and front equation with the $O(\delta)$ terms included.

\subsection{Acoustic zone}

The variable describing the acoustic motion ambient to the hydrodynamic region is

$$
\tilde{\xi}=M \xi
$$

The motion is a longitudinal oscillation and the solution can be written as

$$
(u, \rho, \theta)=\left(U_{ \pm}, R_{ \pm}, \Theta_{ \pm}\right)+\left(u_{a}(\tilde{\xi}, t), M \rho_{a}(\tilde{\xi}, t), M \theta_{a}(\tilde{\xi}, t)\right), \quad p=M^{-1} p_{a}(\tilde{\xi}, t)
$$

where $U_{ \pm}$are the mean velocities behind and in front of the flame respectively. The unsteady field is governed by the linearised acoustic equations. Elimination of $\theta_{a}$ and $\rho_{a}$ among those equations yields the wave equations for the pressure $p_{a}$ and $u_{a}$,

$$
R \frac{\partial^{2} p_{a}}{\partial t^{2}}-\frac{\partial^{2} p_{a}}{\partial \tilde{\xi}^{2}}=0, \quad R \frac{\partial u_{a}}{\partial t}=-\frac{\partial p_{a}}{\partial \tilde{\xi}} .
$$

The acoustic pressure is continuous across the flame, but as will be shown in $\S 3.2$ the flame induces a jump in $u_{a}$, i.e.

$$
\begin{aligned}
& {\left[p_{a}\right]=0 } \\
& {\left[u_{a}\right]=q }\left\{\overline{\left(1+|\nabla F|^{2}\right)^{\frac{1}{2}} \exp \left\{\frac{1}{2} h_{-\infty}\right\}}-1\right\}-\delta q\left\{\frac{l \mathcal{D}}{2} \frac{\partial}{\partial t} \overline{\left(1+|\nabla F|^{2}\right)^{\frac{1}{2}} \exp \left\{-\frac{1}{2} h_{-\infty}\right\}}\right. \\
&\left.+\left(\frac{q}{1+q}+\ln (1+q)\right) \overline{\left(1+|\nabla F|^{2}\right)^{\frac{1}{2}} \frac{\tilde{D}}{\tilde{D} t} \exp \left\{-\frac{1}{2} h_{-\infty}\right\}}\right\}
\end{aligned}
$$

where $\bar{\phi}$ stands for the space average of $\phi$ in the $(\eta, \zeta)$ plane, and $F$ is defined in (3.7) below. At leading order, (3.5) reduces to

$$
\left[u_{a}\right]=q\left\{\overline{\left(1+|\nabla F|^{2}\right)^{\frac{1}{2}} \exp \left\{\frac{1}{2} h_{-\infty}\right\}}-1\right\} .
$$

Obviously, the jump $\left[u_{a}\right]$ acts as an acoustic source, which is explicitly expressed in terms of the flame position and the enthalpy advected to the flame front. The result indicates 
that (a) a wrinkling flame is deemed to generate an acoustic field spontaneously, and (b) enthalpy fluctuations radiate sound waves even when the flame is planar. As will be shown in $\S 3.2$, sound waves in turn exert a leading-order back effect on the flame and hydrodynamics. This is in contrast to typical aeroacoustic problems, where sound waves are merely a passive by-product.

\subsection{Hydrodynamic region}

In the hydrodynamics region, $u_{a}$ and $p_{a, \xi}$ appear spatially uniform on either side of the flame, and can be approximated by their values at $\tilde{\xi}=0^{ \pm}$. In order to facilitate the matching with the solution in the acoustic region, we subtract from the total field the acoustic components as well as the mean background flow by writing (WWMP)

$$
\left.\begin{array}{l}
u=U_{ \pm}+u_{a}\left(0^{ \pm}, t\right)+U, \quad f=F_{a}+F, \\
p=M^{-1} p_{a}(0, t)+P_{ \pm}+p_{a, \tilde{\xi}^{(}\left(0^{ \pm}, t\right)(\xi+F)+P,}
\end{array}\right\}
$$

where $P_{ \pm}$is the mean pressure (with $P_{+}-P_{-}=-q$ ), and $F_{a}^{\prime}=U_{-}-1+u_{a}\left(0^{-}, t\right)$. Let $\mathbf{v}=\mathbf{V}$. Then the hydrodynamic field, to $O(\delta)$ accuracy, satisfies the equations

$$
\left.\begin{array}{c}
\frac{\partial U}{\partial \xi}+\nabla \cdot \mathbf{V}=\frac{\partial \mathbf{V}}{\partial \xi} \cdot \nabla F, \\
R\left[\frac{\partial \tilde{U}}{\partial t}+S \frac{\partial U}{\partial \xi}+\mathbf{V} \cdot \nabla U\right]+[1+R \mathcal{J} h(\xi)] \frac{\partial U}{\partial \xi}=-\frac{\partial P}{\partial \xi}+\delta \operatorname{Pr} \Delta U, \\
R\left[\frac{\partial \mathbf{V}}{\partial t}+S \frac{\partial \mathbf{V}}{\partial \xi}+\mathbf{V} \cdot \nabla \mathbf{V}\right]+[1+R \mathcal{J} h(\xi)] \frac{\partial \mathbf{V}}{\partial \xi}=-\nabla P+\nabla F \frac{\partial P}{\partial \xi}+\delta \operatorname{Pr} \Delta \mathbf{V},
\end{array}\right\}
$$

where $h(\xi)$ is the Heaviside step function, $\mathcal{J}=\left[u_{a}\right]$, and $S=U-F_{t}-\mathbf{V} \cdot \nabla F$. Matching with the outer acoustic solution requires that

$$
U \rightarrow 0, \quad \mathbf{V} \rightarrow 0, \quad P_{\xi} \rightarrow 0 \quad \text { as } \quad \xi \rightarrow \pm \infty
$$

The hydrodynamic motion drives the acoustic motion in the ambient regions by inducing a longitudinal velocity jump. As in WWMP, this key result can be derived by taking the spatial average of the continuity equation in $(3.8)$ in the $(\eta, \zeta)$ plane, and then integrating with respect to $\xi$ to obtain

$$
\bar{U}=\overline{\mathbf{V} \cdot \nabla F}
$$

where the overbar denotes the mentioned average. Taking the limits $\xi \rightarrow 0^{ \pm}$in the first equation in (3.7), and taking the spatial average and using (3.10), we find

$$
\mathcal{J}=\left[u_{a}\right]=-\overline{[[\mathbf{V}]] \cdot \nabla F}+\overline{[[u]]-q}, \quad[[U]]=([[u]]-\overline{[[u]]})+\overline{[[\mathbf{V}]] \cdot \nabla F} .
$$

Inserting the velocity jumps (2.36)-(2.37) into the above equations, we obtain (3.5) and

$$
\begin{aligned}
{[[U]]=} & q\left\{\left(1+|\nabla F|^{2}\right)^{-\frac{1}{2}} \exp \left\{\frac{1}{2} h_{-\infty}\right\}-\overline{\left(1+|\nabla F|^{2}\right)^{\frac{1}{2}} \exp \left\{\frac{1}{2} h_{-\infty}\right\}}\right\} \\
& +\delta\left\{-\frac{q l \mathcal{D}}{2 \kappa}\left[\nabla^{2} F+\nabla \cdot\left(\frac{\kappa}{m} \mathbf{V}^{-}\right)+\frac{\partial}{\partial t}\left(\frac{\kappa}{m}\right)-\kappa \frac{\partial}{\partial t}\left(\frac{\kappa}{m}\right)\right]+\frac{q \chi}{(1+q) m^{2}} \mathbf{A}_{\mathrm{T}} \cdot \nabla F\right. \\
& \left.+\frac{q}{2 \kappa}\left[(\operatorname{Pr}+1) \nabla F \cdot \nabla h_{-\infty}+\left(\frac{q}{1+q}+\ln (1+q)\right)\left(\frac{\kappa}{m} \frac{\tilde{D} h_{-\infty}}{\tilde{D} t}-\kappa \frac{\tilde{D} h_{-\infty}}{m}\right)\right]\right\},
\end{aligned}
$$


where we have put

$$
\mathbf{A}_{\mathrm{T}}=\frac{\tilde{D} \mathbf{V}^{-}}{\tilde{D} t}+\nabla F \frac{\tilde{D} U^{-}}{\tilde{D} t}+\frac{m}{\kappa} \frac{\tilde{D}}{\tilde{D} t} \nabla F+\left(G+u_{a, t}\left(0^{-}, t\right)\right) \nabla F
$$

The transverse velocity jump may be written as

$$
[[\mathbf{V}]]=-[[u]] \nabla F+\delta\left\{\frac{q \chi \kappa}{(1+q) m^{2}} \mathbf{A}_{\mathrm{T}}+\frac{1}{2}(\operatorname{Pr}+1) q \nabla h_{-\infty}\right\},
$$

or alternatively as

$$
\begin{aligned}
{[[\mathbf{V}]]=-[[u]] \nabla F+} & \delta\left\{\operatorname{Pr} \frac{\kappa}{m}\left[\left[\frac{\partial}{\partial \xi}(\mathbf{V}+U \nabla F)\right]\right]+q \operatorname{Pr} \frac{m}{\kappa} \nabla\left(\frac{\kappa}{m}\right)+q \frac{\kappa}{2 m^{2}} \nabla\left(\frac{m^{2}}{\kappa}\right)\right. \\
& \left.+\frac{\kappa}{m^{2}} \ln (1+q) \mathbf{A}_{\mathrm{T}}\right\} .
\end{aligned}
$$

The jump in the pressure $P$ becomes

$$
\begin{aligned}
{[[P]]=} & {\left[R_{+}\left(G+u_{a, t}\left(0^{+}, t\right)\right)-R_{-}\left(G+u_{a, t}\left(0^{-}, t\right)\right)\right] F-q\left[\exp \left\{h_{-\infty}\right\}-1\right]-2 m[[u]] } \\
+ & \delta\left\{\frac{q m}{\kappa} \nabla^{2} F+\left[\frac{\kappa}{m} \frac{\tilde{D} U^{-}}{\tilde{D} t}+\frac{m}{\kappa} \frac{\tilde{D}}{\tilde{D} t}\left(\frac{\kappa}{m}\right)+\left(G+u_{a, t}\left(0^{-}, t\right)\right) \frac{\kappa}{m}\right] \ln (1+q)\right. \\
& \left.\quad-\frac{q m}{2 \kappa^{2}} \nabla F \cdot \nabla \kappa+\frac{q(\operatorname{Pr}+\chi)}{(1+q) m} \mathbf{A}_{\mathrm{T}} \cdot \nabla F+\frac{q m}{\kappa}(\operatorname{Pr}+1) \nabla F \cdot \nabla h_{-\infty}\right\} .
\end{aligned}
$$

Note that the local acoustic acceleration, $u_{a, t}\left(0^{ \pm}, t\right)$, plays the same role as gravity $G$ throughout (3.14)-(3.16), suggesting that the acoustic field creates an unsteady RayleighTaylor effect, through which it acts on the flame.

The equation governing the advection of enthalpy $h \equiv H$, to $O(\delta)$ accuracy, reads

$$
R\left[\frac{\partial H}{\partial t}+S \frac{\partial H}{\partial \xi}+\mathbf{V} \cdot \nabla H\right]+[1+R \mathcal{J} h(\xi)] \frac{\partial H}{\partial \xi}=\delta \Delta H,
$$

which is subject to the initial condition and jump condition (see (2.24) and (2.29))

$$
\left.\begin{array}{rl}
H & \rightarrow H_{-\infty}(\eta, \zeta, t-\xi) \quad \text { as } \quad \xi \rightarrow-\infty \\
[H]] & =\delta\left\{(\ln (1+q)+q /(1+q)) \frac{\kappa}{m_{0}^{2}} \frac{\tilde{D}}{\tilde{D} t} h_{-\infty}-\frac{l \mathcal{D}}{m_{0}} \Gamma\right\} .
\end{array}\right\}
$$

The system (3.17)-(3.18) has to be solved to obtain $h_{-\infty} \equiv H(0, \eta, \zeta, t)$.

The specification of $H_{-\infty}$ depends on where enthalpy fluctuations are introduced. If they are introduced just upstream the hydrodynamic zone, i.e. at a location where $1 \ll(-\xi) \ll L / M$, then $H_{-\infty}=\mathcal{H}_{I}$, where $\mathcal{H}_{I}$ characterises the spatial distribution and temporal modulation of the enthalpy imposed. However, if enthalpy fluctuations are imposed at the inlet, then determination of $\mathcal{H}_{-\infty}$ requires us to consider the enthalpy advection in the upstream acoustic region, where $H$ is governed, to leading order, by

$$
\frac{\partial H}{\partial t}+M\left(U_{-}+u_{a}(\tilde{\xi}, t)-f_{t}\right) \frac{\partial H}{\partial \tilde{\xi}}=0 .
$$

Clearly, the advection is influenced by sound waves produced by the flame. On using the method of characteristics, the solution, subjected to an error of $O(M)$, can be written as

$$
H=H_{-\infty}\left(t+\int^{t}\left[u_{a}(\tilde{\xi}, \tau)-u_{a}(0, \tau)\right] \mathrm{d} \tau-\tilde{\xi} / M-F\right) .
$$




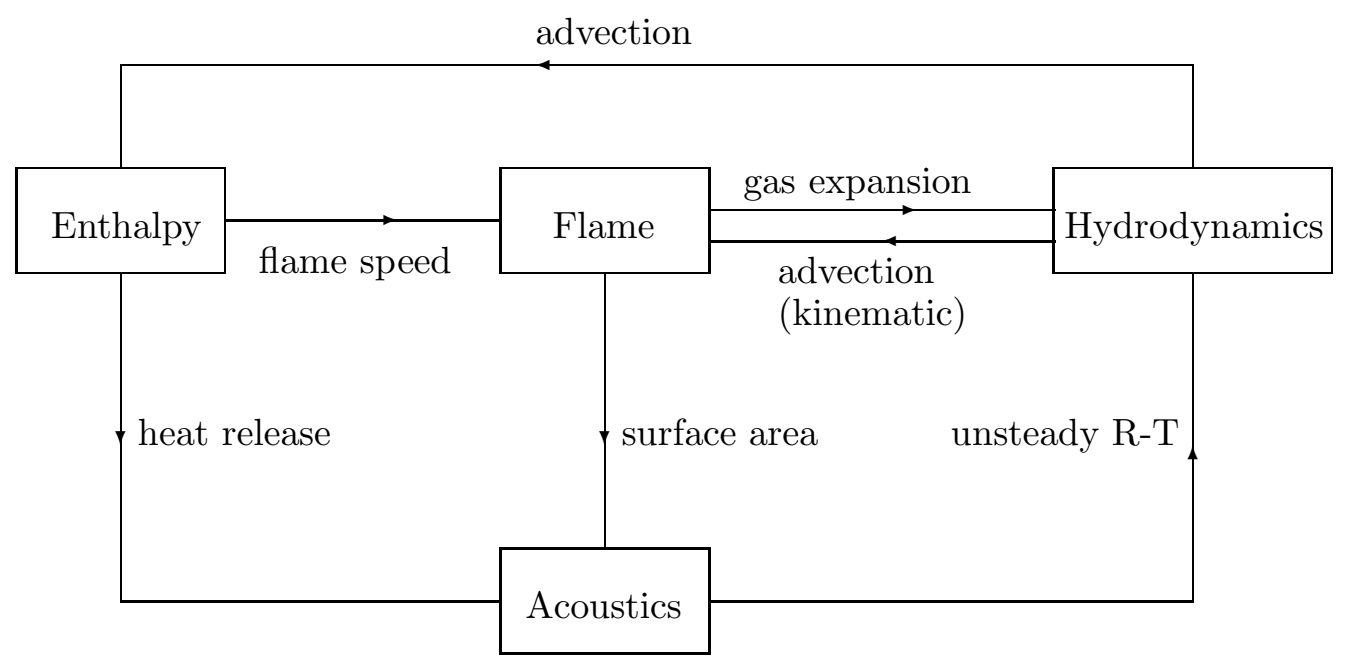

Figure 1: A diagrammatic illustration of the couplings among the flame, hydrodynamics, acoustics and enthalpy perturbations.

Applying the boundary condition at the inlet of the duct, $\tilde{\xi}=-\sigma L$, yields

$$
H_{-\infty}\left(\eta, \zeta, t+\int^{t}\left[u_{a}(-\sigma L, \tau)-u_{a}(0, \tau)\right] \mathrm{d} \tau+\sigma L / M-F\right)=\mathcal{H}_{I}(\eta, \zeta, t) .
$$

On approaching the hydrodynamic region, $\tilde{\xi}=O(M), H \sim H_{-\infty}(t-\xi-F)$. Determination of $H_{-\infty}$ explicitly in terms of $\mathcal{H}_{I}$ is however not straightforward, because $H_{-\infty}$ contains $u_{a}$, which in turn depends on $H_{-\infty}$ (as a function of $t$ ). Such an interdependence reflects the long-range upstream influence of the flame through the spontaneously emitted sound waves. In many practical situations, enthalpy fluctuations present at the inlet may arise due to the acoustic pressure interfering with the mixture supply, in which case $\mathcal{H}_{I}$ may also be a functional of $p_{a}$ or $u_{a}$, the precise relation depending on the dynamics of the mixing/feeding system.

The coupling with the flame is completed through the front equation,

$$
F_{t}+\mathbf{V}^{-} \cdot \nabla F=U^{-}-(m-1)+\delta\left\{\mathrm{M}_{a} \Gamma-\frac{\kappa}{2 m}\left[\ln (1+q)+\frac{q}{1+q}\right] \frac{\tilde{D} h_{-\infty}}{\tilde{D} t}\right\} .
$$

Equations (3.8), (3.17) and (3.22), coupled to the acoustic equations (3.3) via (3.5), form an overall interactive system to describe the complex flame-acoustic-enthalpy coupling. As is illustrated in figure 1, upstream enthalpy fluctuations are advected by the hydrodynamic field of the flame. On the other hand, enthalpy fluctuations directly influence chemical reactions and therefore modify flame speed, through which the hydrodynamics is simultaneously influenced by enthalpy fluctuations. Moreover, the flame motion and enthalpy fluctuations radiate sound waves, whose acceleration creates a $\mathrm{R}-\mathrm{T}$ effect to act on the hydrodynamics and ultimately on the flame.

The reduced system remains highly nonlinear, and in general has to be solved numerically. Nevertheless, analytical progress may be made in a few special cases, where generally coupled fundamental processes of combustion instability become decoupled. We now consider several of those processes, and our focus will be on new physical mechanisms which operate only when enthalpy fluctuations are present. 


\section{Linear response to small-amplitude enthalpy fluctuation}

Enthalpy fluctuations in two or three-dimensions interact with the flame to generate a hydrodynamic motion. This is an important effect, which has been addressed theoretically in the literature. We shall demonstrate it by calculating the flame response to smallamplitude enthalpy fluctuations in the same spirit as Searby \& Clavin (1986) and Aldredge \& Williams (1991) did for vortical disturbances. The flame is assumed to be intrinsically stable. Then in response to an imposed fluctuation, the flame is slightly wrinkled and the induced hydrodynamic motion is weak, so that (3.8) and the jump conditions can be linearized. Since the spontaneously generated sound waves are negligible, the enthalpy disturbance is simply advected by the background mean flow.

An enthalpy fluctuation in general has a continuous spectrum, and may be represented by an integral over all Fourier modes. Owing to linearity, it suffices to consider only a single component,

$$
H_{\mathcal{I}}=h_{\mathcal{I}} \mathrm{e}^{\mathrm{i}\left(\omega \xi+k_{2} \eta+k_{3} \zeta-\omega t\right)},
$$

imposed at $\xi_{\mathcal{I}}$ with $-\xi_{\mathcal{I}} \ll L / M$, where $\mathbf{k}^{\dagger} \equiv\left(k_{2}, k_{3}\right)$. The linearised advection equation (3.17) can be solved to obtain the enthalpy at the flame front

$$
h_{-\infty}(\eta, \zeta, t)=H(0, \eta, \zeta, t)=h_{\mathcal{I}} \mathrm{e}^{\left(k^{2}+\omega^{2}\right) \delta \xi_{\mathcal{I}}} \mathrm{e}^{\mathrm{i}\left(k_{2} \eta+k_{3} \zeta-\omega t\right)} \equiv \hat{h}_{-\infty} \mathrm{e}^{\mathrm{i}\left(k_{2} \eta+k_{3} \zeta-\omega t\right)},
$$

where $k=\left(k_{2}^{2}+k_{3}^{2}\right)^{\frac{1}{2}}$, and $\hat{h}_{-\infty}$ is related to $h_{\mathcal{I}}$ via the relation $\hat{h}_{-\infty}=h_{\mathcal{I}} \mathrm{e}^{\left(k^{2}+\omega^{2}\right) \delta \xi_{\mathcal{I}}}$, which accounts for attenuation of the enthalpy fluctuation.

The steady-state solution for $(U, \mathbf{V}, P)$ and $F$ can be written as

$$
(U, \mathbf{V}, P, F)=(\bar{U}, \overline{\mathbf{V}}, \bar{P}, \bar{F}) \mathrm{e}^{\mathrm{i}\left(k_{2} \eta+k_{3} \zeta-\omega t\right)} .
$$

After substituting this into the linearised (3.8), it is easy to find that

$$
\bar{P}=P^{ \pm} \mathrm{e}^{\mp k \xi}, \quad \bar{U}=\frac{\mp k P^{ \pm} \mathrm{e}^{\mp k \xi}}{\mathrm{i} R_{ \pm} \omega \pm k}+C^{ \pm} \mathrm{e}^{\mathrm{i} \mathcal{S}_{ \pm} \xi}, \quad \overline{\mathbf{V}}=\frac{\mathrm{i} \mathbf{k}^{\dagger} P^{ \pm} \mathrm{e}^{\mp k \xi}}{\mathrm{i} R_{ \pm} \omega \pm k}+\mathbf{D}^{ \pm} \mathrm{e}^{\mathrm{i} \mathcal{S}_{ \pm} \xi}
$$

where $\mathcal{S}_{ \pm}=\omega R_{ \pm}+\mathrm{i} \operatorname{Pr}\left(k^{2}+\omega^{2} R_{ \pm}^{2}\right) \delta+O\left(\delta^{2}\right)$, and the constants $C^{ \pm} \& \mathbf{D}^{ \pm}$correspond to vortical fluctuations. To focus on enthalpy fluctuations, we assumed that vortical fluctuations are absent in the upstream, i.e. $C^{-}=\mathbf{D}^{-}=0$. However, $C^{+}$and $\mathbf{D}^{+}$are non-zero, that is, enthalpy fluctuations, by interacting with the flame, generates vorticity. Substitution of (4.1)-(4.2) into the continuity equation, linearised front equation and jump conditions leads to a system of 6 simultaneous equations, solving which we obtain

$$
\begin{gathered}
\bar{F}=\frac{[(q+1) k-\mathrm{i} \omega]+\delta Q_{F}(k, \omega)}{\Delta(k, \omega)} \hat{h}_{-\infty}, \\
\bar{U}\left(0^{-}, \mathbf{k}^{\dagger}\right)=\left\{\frac{-(q+1) \mathrm{i} \omega k-\omega^{2}+\delta Q_{u}(k, \omega)}{\Delta(k, \omega)}+\frac{1}{2}\left(1+\delta Q_{h}\right)\right\} \hat{h}_{-\infty},
\end{gathered}
$$

where

$$
\Delta(k, \omega)=\mathcal{A} \omega^{2}+\mathrm{i} \mathcal{B} \omega-\mathcal{C},
$$

with the constants $\mathcal{A}, \mathcal{B}$ and $\mathcal{C}$ being the same as given in Wu \& Law (2009), while the rather unwieldy expressions for $Q_{F}, Q_{u}$ and $Q_{h}$ can be found in Wu \& Moin (2008). To leading order, one may put $Q_{F}=Q_{u}=Q_{h}=0$, and

$$
\Delta(k, \omega) \approx\left(R_{+}+R_{-}\right) \omega^{2}+2 \mathrm{i} k \omega+q k^{2}+k\left(R_{+}-R_{-}\right) G .
$$



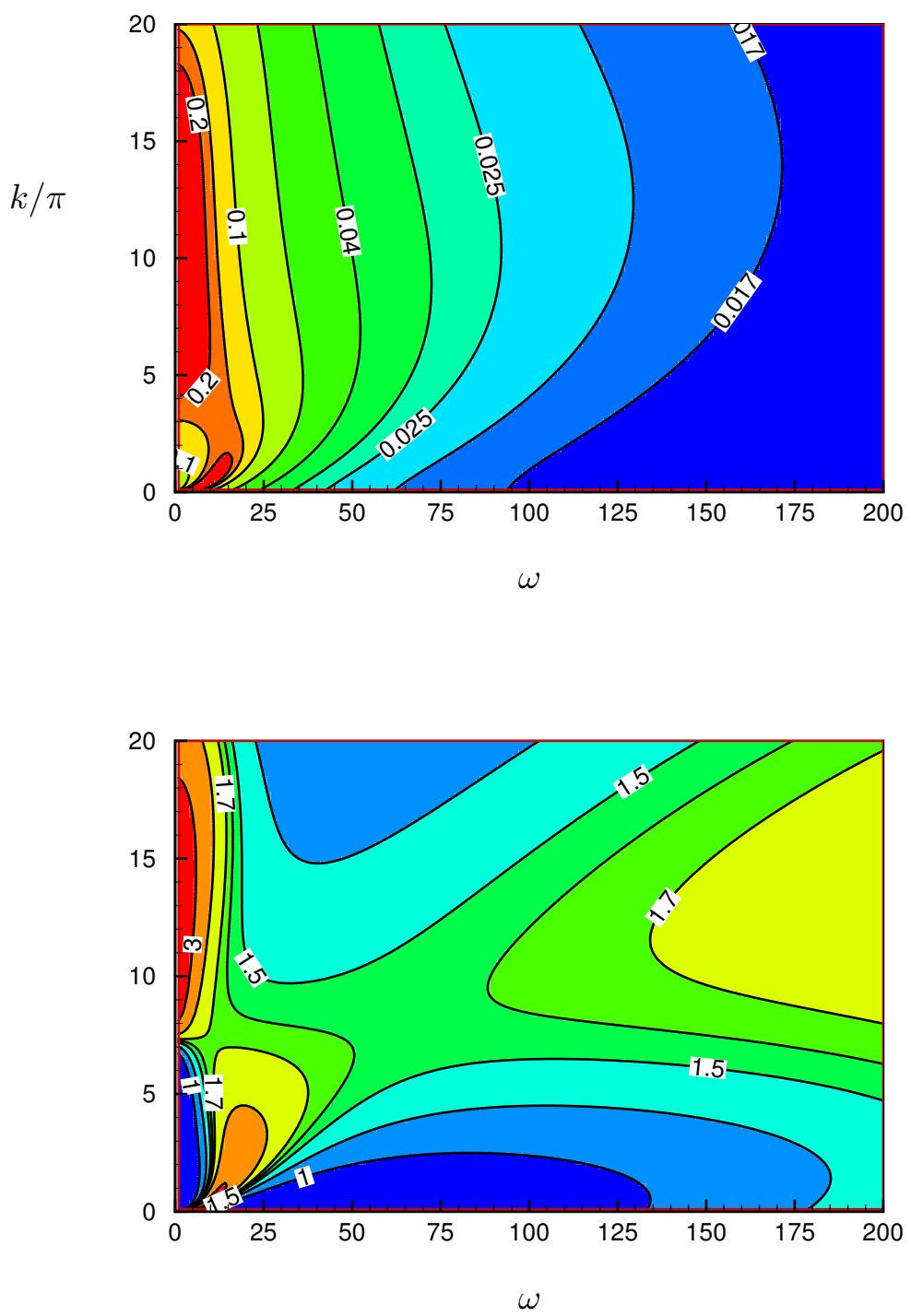

Figure 2: Contours of transfer functions $|F(k, \omega)| / \hat{h}_{-\infty}$ and $\left|U_{-}(0, k, \omega)\right| / \hat{h}_{-\infty}$. 


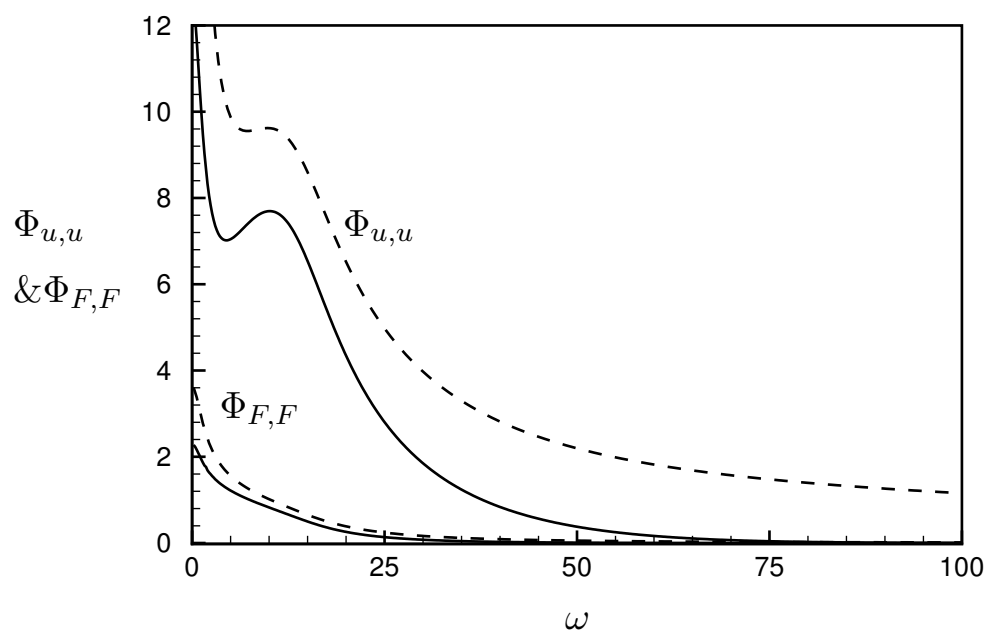

Figure 3: Spectra $\Phi_{u, u}$ and $\Phi_{F, F}$. Solid lines: $\xi_{\mathcal{I}}=-0.5$; dashed lines: $\xi_{\mathcal{I}}=0$.

If the fluctuation is for simplicity assumed to be homogeneous and isotropic, then its spectrum $\Phi(\mathbf{k})$ can be written as $\Phi(|\mathbf{k}|)$, a function of $|\mathbf{k}|=\left(k_{2}^{2}+k_{3}^{2}+\omega^{2}\right)^{\frac{1}{2}}$, where $\mathbf{k}=\left(\omega, \mathbf{k}^{\dagger}\right)$. The spectra of the fluctuations in the velocity at the front and the flame position, $\Phi_{u, u}(\omega)$ and $\Phi_{F, F}(\omega)$, can be expressed as

$$
\begin{aligned}
& \Phi_{u, u}(\omega)=\int_{-\infty}^{\infty}\left|\frac{(q+1) \mathrm{i} \omega k+\omega^{2}-\delta Q_{u}}{\Delta(k, \omega)}-\frac{1}{2}\left(1+\delta Q_{h}\right)\right|^{2} \mathrm{e}^{2\left(k^{2}+\omega^{2}\right) \delta \xi_{\mathcal{I}}} \Phi(|\mathbf{k}|) \mathrm{d} \mathbf{k}^{\dagger}, \\
& \Phi_{F, F}(\omega)=\int_{-\infty}^{\infty}\left|\frac{(q+1) k-\mathrm{i} \omega+\delta Q_{F}}{\Delta(k, \omega)}\right|^{2} \mathrm{e}^{2\left(k^{2}+\omega^{2}\right) \delta \xi_{\mathcal{I}}} \Phi(|\mathbf{k}|) \mathrm{d} \mathbf{k}^{\dagger}
\end{aligned}
$$

The transfer functions $|F(k, \omega)| / \hat{h}_{-\infty}$ and $\left|U_{-}(k, \omega)\right| / \hat{h}_{-\infty}$, which measure the sensitivity of flame-front displacement and fluid motion to enthalpy perturbations, are calculated by using (4.3)-(4.4), and the parameters used are as follows

$$
\begin{gathered}
h^{*}=10 \mathrm{~cm}, \quad l^{*}=120 \mathrm{~cm}, \quad q=5, \quad U_{L}=10 \mathrm{cms}^{-1}, \\
D_{t h}^{*}=0.22 \mathrm{~cm}^{2} \mathrm{~s}^{-1}, \quad a^{*}=340 \mathrm{~ms}^{-1}, \quad \text { Le }=1.11, \quad \beta=12, \quad \gamma=1.4 .
\end{gathered}
$$

They give rise to $\delta=2.2 \times 10^{-3}$, Mach number $M=2.94 \times 10^{-4}$ and Markstein number $M a=4$. The same set of parameters will be used in the rest of the paper unless stated otherwise. The contour plots displayed in Fig.2 indicate that the typical values of $|F| / \hat{h}_{-\infty}$ and $\left|U_{-}\right| / \hat{h}_{-\infty}$ are about 0.02 and 1.5 respectively. The result suggests that in the presence of $1.6 \%$ local mass-fraction fluctuation at the flame front (which corresponds to $\hat{h}_{-\infty}=1$ ), the wrinkled flame has a $2 \mathrm{~mm}$ flame-brush thickness, and the induced hydrodynamic velocity becomes comparable with the laminar flame speed.

Spectra of enthalpy fluctuations are unavailable in the literature. We assume that they are similar to those for scalar fluctuations, and so take

$$
\Phi(|\mathbf{k}|)=\overline{h^{2}}\left(1+\left(r_{t}|\mathbf{k}|\right)^{2}\right)^{-\frac{11}{6}},
$$

which characterizes temperature fluctuations in a uniform mean flow (see (3-182) and (3221) in Hinze 1959); here $r_{t}$ the ratio of the integral length scale to $h^{*}$. In the calculation, 


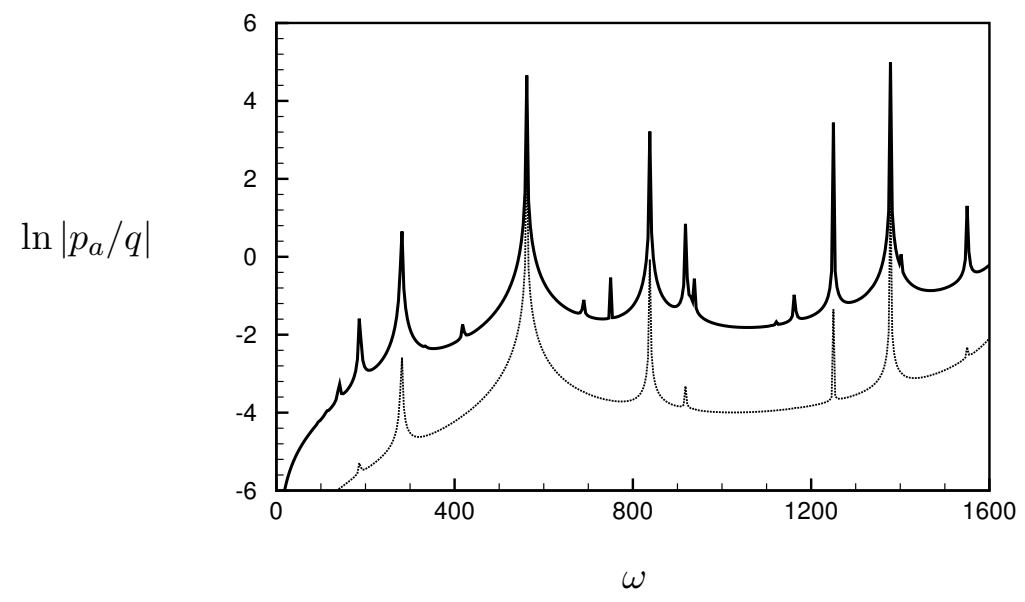

Figure 4: The intensity of the sound waves emitted by enthalpy fluctuation (5.2) v.s. $\omega$ for $\hat{h}=1$ (solid lines) and $\hat{h}=0.2$ (dotted lines). The mean flame position corresponds to $\sigma=0.75$.

we take $r_{t}=0.1$. The result is shown in Fig.3. The spectrum of the flame brush, $\Phi_{F, F}$, decays monotonically with $\omega$, but $\Phi_{u, u}$ exhibits a peak at a quite low frequency. Both attenuate rapidly as $\omega$ increases. The attenuation is controlled by $\xi_{\mathcal{I}}$ (taken to be -0.5 ), and is eliminated if $\xi_{\mathcal{I}}=0$. The spectra in this limit case serve as the upper bounds, and are included for comparison. The overall qualitative feature is not altered by $\xi_{\mathcal{I}}$.

Due to the wrinkling effect, the change in the flame surface area (or length in the twodimensional case) must be proportional to the enthalpy intensity squared. This scaling behaviour is consistent with the DNS result of Garrido-Lopez \& Sarkar 2005).

\section{Acoustic radiation of enthalpy fluctuations}

As enthalpy fluctuations induce a hydrodynamic field causing a planar flame to wrinkle, sound waves are emitted due to the change of the flame-surface area. The resulting acoustic field is inherently coupled with the hydrodynamics, the flame motion and enthalpy advection in a strongly nonlinear fashion for two- or three-dimensional enthalpy fluctuations with $O(1)$ values of $\mathcal{H}_{I}$ or $h_{-\infty}$. In the rest of this paper, we will consider the case where the upstream enthalpy fluctuation is one-dimensional, as was assumed in earlier studies (Lieuwen et al. 2001, Birbaud et al. 2008). Further analytical progress is then possible even for order-one $\mathcal{H}_{I}$. The front equation admits a simple solution,

$$
F=\bar{F}=\int^{t}\left\{1-\exp \left(\frac{1}{2} h_{-\infty}(t)\right)\right\} \mathrm{d} t
$$

representing a vibratory planar flame, for which the hydrodynamics field is absent. For simplicity, the enthalpy is assumed to be modulated periodically in time at a location $-\tilde{\xi} \ll L M^{-1}$, and so we may take

$$
h_{-\infty}=\mathcal{H}_{I}(0, t)=\widehat{h}\left(\mathrm{e}^{\mathrm{i} \omega t}+\text { c.c. }\right) .
$$


The jump across the hydrodynamic zone, (3.6), can be written as a Fourier series,

$$
\left[u_{a}\right]=q\left\{\exp \left\{\frac{1}{2} \widehat{h}\left(\mathrm{e}^{\mathrm{i} \omega t}+\text { c.c. }\right)\right\}-1\right\}=\sum j_{n} \mathrm{e}^{\mathrm{i} n \omega t} \text { with } j_{n}=q \hat{h}^{n} / n !
$$

and it indicates that a one-dimensional enthalpy fluctuation produces sound waves.

\subsection{Non-resonant case}

Assume that none of the harmonics in the enthalpy fluctuation has a frequency coinciding with any eigen frequency of the duct modes. Then the solution for the acoustic field can be expressed as Fourier series

$$
\left(p_{a}, u_{a}\right)=\sum\left\{\left(1, R_{ \pm}^{-\frac{1}{2}}\right) a_{r}^{ \pm} \mathrm{e}^{-\mathrm{i} R_{ \pm}^{\frac{1}{2}} n \omega \tilde{\xi}}+\left(1,-R_{ \pm}^{-\frac{1}{2}}\right) a_{l}^{ \pm} \mathrm{e}^{\mathrm{i} R_{ \pm}^{\frac{1}{2}} n \omega \tilde{\xi}}\right\} \mathrm{e}^{\mathrm{i} n \omega t}+c . c .
$$

The constants $a_{l}^{ \pm}$and $a_{r}^{ \pm}$can be determined by using the jump conditions (3.4)-(3.5), and the boundary conditions the two ends of the duct, namely, $u_{a}=0$ at $\tilde{\xi}=-\sigma L$ and $p_{a}=0$ at $\tilde{\xi}=(1-\sigma) L$, where $L$ is related to the dimensional length of the duct $l^{*}$ via $L=M l^{*} / h^{*}$, and $\sigma$ is a parameter characterising the mean flame position. We find that

$$
\left(a_{l}^{-}, a_{r}^{-}\right)=-\frac{\mathrm{i} j_{n} R_{+}^{\frac{1}{2}} \tan \left(R_{+}^{\frac{1}{2}}(1-\sigma) n \omega L\right)}{2 \Delta_{s}(n \omega ; \sigma) \cos \left(R_{-}^{\frac{1}{2}} \sigma n \omega L\right)}\left(\mathrm{e}^{\mathrm{i} R_{-}^{\frac{1}{2}} \sigma n \omega L}, \mathrm{e}^{-\mathrm{i} R_{-}^{\frac{1}{2}} \sigma n \omega L}\right),
$$

and the acoustic pressure level at the entrance (which we take to represent the sound field)

$$
\left|p_{a}\right|=\sum_{n=1}^{\infty} \frac{R_{+}^{\frac{1}{2}} j_{n} \tan \left(R_{+}^{\frac{1}{2}}(1-\sigma) n \omega L\right)}{\Delta_{s}(n \omega ; \sigma) \cos \left(R_{-}^{\frac{1}{2}} \sigma n \omega L\right)},
$$

where

$$
\Delta_{s}(\omega ; \sigma)=\left(\frac{R_{+}}{R_{-}}\right)^{\frac{1}{2}} \tan \left(R_{-}^{\frac{1}{2}} \sigma \omega L\right) \tan \left(R_{+}^{\frac{1}{2}}(1-\sigma) \omega L\right)-1 .
$$

The characteristic acoustic frequencies of the duct are given by the roots of the acoustic dispersion relation,

$$
\Delta_{s}(\omega ; \sigma)=0
$$

A countable number of roots $\omega_{k}=\omega_{k}(\sigma)(k=1,2, \ldots)$ may exist.

Fig.4 shows the variation of the acoustic wave intensity with the frequency of the enthalpy fluctuation for a planar flame with its mean position at $0.75 \mathrm{~L}$ distance from the inlet. A spiky appearance is observed. Discrete 'peaks' appear at resonant frequencies, which satisfy the relation

$$
n \omega=\omega_{k},
$$

where $n \neq 0$ is an arbitrary integer. Since the effective forcing $j_{n}$ at the $\mathrm{n}$-th harmonic frequency $n \omega$ behaves as $j_{n}=\hat{h}^{n} / n$ !, 'peaks' at larger values of $n$ are weaker, and for small $\hat{h}$ they almost disappear so that the dotted curves (for $\hat{h}=0.2$ ) become less spiky. 


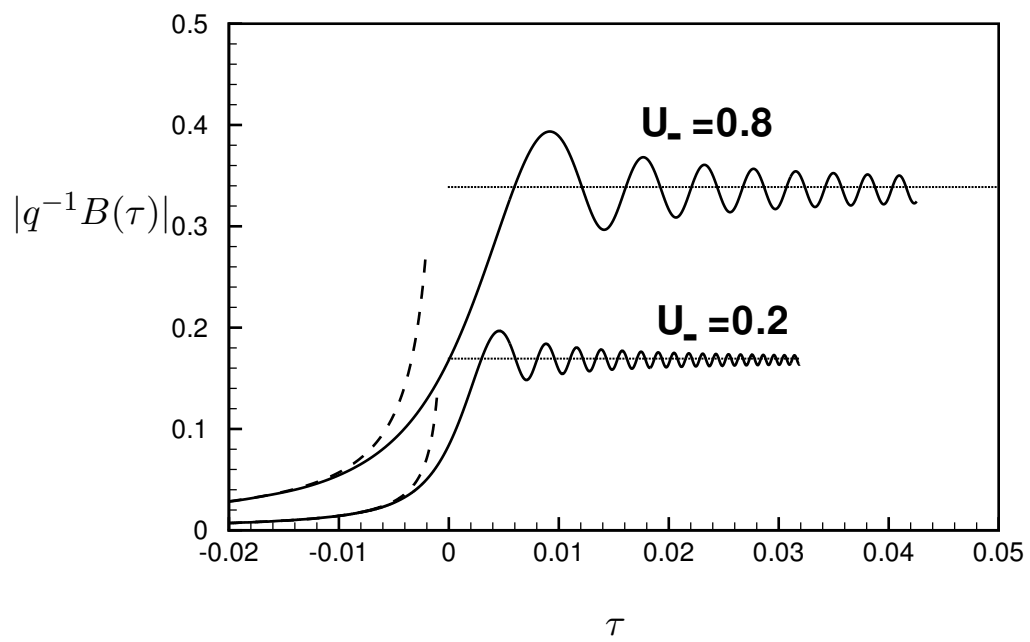

Figure 5: Evolution of the sound intensity through the transient resonant phase for $U_{-}=0.2$ and 0.8 . The dashed and dotted lines represent the asymptotic upstream and downstream behaviours, (5.16) and (5.17), respectively.

\subsection{Discussion of resonance}

For a flame with a fixed mean position, the stationary solution (5.5) becomes invalid when the resonance condition (5.8) holds. Instead the sound wave would amplify in proportion to time $t$. If the acoustic loss is sufficiently small, the sound wave may amplify to such an extend that it acts back on the flame to cause parametric instability. The instability will in turn produce extra sound waves.

If a flame is moving, then for a fixed $\omega$, resonance is of transient nature in that it takes place only in a vicinity of the position corresponding to $\sigma_{c}$ for which $n \omega=\omega_{k}\left(\sigma_{c}\right)$. For definitiveness, suppose that at time $t=0$, a flame is positioned at the entrance. Then at an arbitrary time $t$, its mean position can be written as $M\left(1-U_{-}\right) t=(1-\sigma) L$. The flame moves to the resonance position $\left(1-\sigma_{c}\right) L$ at $t_{c}=\left(1-\sigma_{c}\right) L /\left(M\left(1-U_{-}\right)\right)$. When $t$ (or $\sigma$ ) is sufficiently different from $t_{c}$ (or $\sigma_{c}$ ), the acoustic response is of quasi-steady and non-resonant nature, and its solution is given by (5.4), in which $\sigma$ plays the role of a parameter. However, as the flame approaches the resonant location, i.e. as $\sigma \rightarrow \sigma_{c}$,

$$
a_{l}^{-} \mathrm{e}^{-\mathrm{i} R_{-}^{\frac{1}{2}} \sigma_{c} \omega L} \rightarrow \frac{\mathrm{i} j_{n}\left(1-R_{+} / R_{-}\right)^{-1}}{2 \tan \left(R_{-}^{\frac{1}{2}} \sigma_{c} \omega L\right) \sin \left(R_{-}^{\frac{1}{2}} \sigma_{c} \omega L\right)}\left(\omega\left(1-U_{-}\right) M\left(t-t_{c}\right)\right)^{-1} .
$$

The quasi-steady response is no longer valid when $M\left(t-t_{c}\right)=O\left(M^{1 / 2}\right)$ because the rate of the change of the response becomes comparable with the rate of change of the mean flame position. To describe the evolution of the sound in the resonant region, we introduce

$$
t=t_{c}+M^{-\frac{1}{2}} \tau
$$

Relations (5.9)-(5.10) imply that in the resonant region, the acoustic pressure and 
velocity are of $O\left(M^{-\frac{1}{2}} h_{-\infty}\right)$. We assume that $h_{-\infty} \sim M^{\frac{1}{2}}$ so that the solution expands as

$$
p_{a}=B(\tau) p_{a, 1}+M^{\frac{1}{2}} p_{a, 2}+\ldots, \quad u_{a}=B(\tau) u_{a, 1}+M^{\frac{1}{2}} u_{a, 2}+\ldots,
$$

where $B$ is the amplitude function of the acoustic mode of the duct

$$
\left(p_{a, 1}, u_{a, 1}\right)=\left\{\left(1, R_{ \pm}^{-\frac{1}{2}}\right) a_{r}^{ \pm} \mathrm{e}^{-\mathrm{i} R_{ \pm}^{\frac{1}{2}} \omega \tilde{\xi}}+\left(1,-R_{ \pm}^{-\frac{1}{2}}\right) a_{l}^{ \pm} \mathrm{e}^{\mathrm{i} R_{ \pm}^{\frac{1}{2}} \omega \tilde{\xi}}\right\} \mathrm{e}^{\mathrm{i} \omega t}
$$

The eigenfunction is normalized such that $a_{l}^{-}=\mathrm{e}^{2 \mathrm{i} R_{-}^{\frac{1}{2}} \sigma \omega L}$. Inserting (5.11) into (3.3), then at $O\left(M^{\frac{1}{2}}\right)$ we have

$$
R \frac{\partial^{2} p_{a, 2}}{\partial t^{2}}-\frac{\partial^{2} p_{a, 2}}{\partial \tilde{\xi}^{2}}=-2 R \mathrm{i} \omega B^{\prime}(\tau) p_{a, 1}, \quad R \frac{\partial u_{a, 2}}{\partial t}=-\frac{\partial p_{a, 2}}{\partial \tilde{\xi}}-R B^{\prime}(\tau) u_{a, 1} .
$$

Since the homogeneous equations admit an eigen solution, the inhomogeneous system has an acceptable solution only when a solvability condition is satisfied. The latter leads to the amplitude equation for the acoustic mode (Wu \& Moin 2008)

$$
B^{\prime}=\mathrm{i} \chi_{s} \tau B+N
$$

where $\chi_{s}=(\omega / L)\left(1-R_{+} / R_{-}\right)\left(1-U_{-}\right) \tan \left(R_{-}^{\frac{1}{2}} \sigma_{c} \omega L\right) \Lambda$, and $N=j \Lambda /\left(2 L \sin \left(R_{-}^{\frac{1}{2}} \sigma_{c} \omega L\right)\right)$ with $\Lambda$ being the same as (4.41) of WWMP. The relevant solution to (5.14) is found as

$$
B=N \mathrm{e}^{\mathrm{i} \chi_{s} \tau^{2} / 2} \int_{-\infty}^{\tau} \mathrm{e}^{-\mathrm{i} \chi_{s} \tau^{2} / 2} \mathrm{~d} \tau .
$$

Note that as $\tau \rightarrow-\infty$,

$$
\left.B \rightarrow \mathrm{i}\left(1-R_{+} / R_{-}\right)^{-1} j /\left(2 \tan \left(R_{-}^{\frac{1}{2}} \sigma_{c} \omega L\right) \sin \left(R_{-}^{\frac{1}{2}} \sigma_{c} \omega L\right)\right)\right)\left(\omega\left(1-U_{-}\right)\right)^{-1},
$$

matching to the pre-resonance solution (5.9) as expected, while as $\tau \rightarrow \infty$,

$$
B \rightarrow B_{\infty} \mathrm{e}^{\mathrm{i} \chi_{s} \tau^{2} / 2} \quad \text { with } \quad B_{\infty}=N \int_{0}^{\infty} \frac{\mathrm{e}^{-\mathrm{i} \chi_{s} \tau / 2}}{\sqrt{\tau}} \mathrm{d} \tau .
$$

Fig.5 shows that the sound intensity may amplify considerably via the transient resonance during the time window in which the flame propagates through the resonant location. The gain in the intensity depends on $\left(1-U_{-}\right)$, the propagation speed relative to the duct; it decreases with the feeding velocity $U_{-}$of the oncoming mixture. Interestingly, the acoustic amplitude in the post-resonance phase is oscillatory.

\section{Parametric instability of the flame: linear analysis}

Enthalpy fluctuations and the sound that they generate, exert a periodic forcing on the flame. This leads to a parametric instability of the planar flame (5.1), as was shown in an earlier preliminary study (Wu 2005). In this section, we present an analysis based on the leading-order approximation of the hydrodynamic equations and the jump conditions. A refined, but more involved, analysis with $O(\delta)$ accuracy is given in the appendix. 
The planar flame (5.1) is perturbed so that

$$
F=\int^{t}\left\{1-\exp \left(\frac{1}{2} h_{-\infty}(t)\right)\right\} \mathrm{d} t+\tilde{F} .
$$

For $\tilde{F} \ll 1$, the hydrodynamic motion is weak, governed by linearized (3.8)

$$
\frac{\partial U}{\partial \xi}+\nabla \cdot \mathbf{V}=0, \quad R \frac{\partial U}{\partial t}+I_{0}(t) \frac{\partial U}{\partial \xi}=-\frac{\partial P}{\partial \xi}, \quad R \frac{\partial \mathbf{V}}{\partial t}+I_{0}(t) \frac{\partial \mathbf{V}}{\partial \xi}=-\nabla P
$$

where we have put

$$
I_{0}(t)=\exp \left\{\frac{1}{2} h_{-\infty}(t)\right\}
$$

Since the coefficients of equations (6.2) are independent of the transverse variables, one may seek solutions of the normal-mode form,

$$
(\tilde{F}, P, U, \mathbf{V})=(\alpha(t), \tilde{P}(\xi, t), \tilde{U}(\xi, t), \tilde{\mathbf{V}}(\xi, t)) \mathrm{e}^{\mathrm{i}\left(k_{2} \eta+k_{3} \zeta\right)}
$$

It is found that (cf. Wu \& Law 2009)

$$
\begin{aligned}
(\tilde{U}, \tilde{\mathbf{V}}) & = \begin{cases}\left(1, \mathrm{i} \mathbf{k}^{\dagger} / k\right) \phi_{-}(t) \mathrm{e}^{k \xi} & \xi<0, \\
\left(1,-\mathrm{i} \mathbf{k}^{\dagger} / k\right) \phi_{+}(t) \mathrm{e}^{-k \xi}+\left(C_{+}(\tilde{t}), D_{+}(\tilde{t})\right) & \xi>0,\end{cases} \\
\tilde{P} & = \begin{cases}-k^{-1}\left(R_{-} \phi_{-}^{\prime}+k I_{0} \phi_{-}\right) \mathrm{e}^{k \tilde{\xi}} & \xi<0, \\
k^{-1}\left(R_{+} \phi_{+}^{\prime}-k I_{0} \phi_{+}\right) \mathrm{e}^{-k \tilde{\xi}} & \tilde{\xi}>0,\end{cases}
\end{aligned}
$$

where $\phi_{ \pm}$are functions of $t$, while $C_{+}$and $\mathbf{D}_{+}$are arbitrary functions of $\tilde{t} \equiv \int^{t} I_{0}(\tau) \mathrm{d} \tau-$ $R_{+} \xi$. It follows from the continuity equation that

$$
-R_{+} C_{+}^{\prime}+\mathrm{i} \mathbf{k}^{\dagger} \cdot \mathbf{D}_{+}=0
$$

while the linearised jump conditions and front equation, $\tilde{F}_{t}=U\left(0^{-}, \eta, \zeta\right)$, give the relations

$$
\left.\begin{array}{rl}
\left(R_{+} \phi_{+}^{\prime}-k I_{0} \phi_{+}\right) & =-\left(R_{-} \phi_{-}^{\prime}+k I_{0} \phi_{-}\right)+k\left\{\left(R_{+}-R_{-}\right) G-\Delta p_{a, \xi}\right\} \alpha, \\
-\frac{\mathrm{i} \mathbf{k}^{\dagger}}{k} \phi_{+}+\mathbf{D}_{+} & =\frac{\mathrm{i} \mathbf{k}^{\dagger}}{k} \phi_{-}-\mathrm{i} \mathbf{k}^{\dagger} q I_{0} \alpha, \quad \phi_{+}+C_{+}=\phi_{-}, \quad \alpha^{\prime}=\phi_{-} \cdot
\end{array}\right\}
$$

After eliminating $\phi_{ \pm}, C_{+}$and $\mathbf{D}_{+}$, we obtain

$$
\left(R_{+}+R_{-}\right) \alpha^{\prime \prime}(t)+2 k I_{0}(t) \alpha^{\prime}(t)-\left\{q k^{2} I_{0}^{2}(t)+k\left(\left(R_{+}-R_{-}\right) G-\Delta p_{a, \xi}(t)\right)\right\} \alpha=0,
$$

which is a parametrically excited (Floquet) system. In the appendix, this equation is generalised to (A.16), which has $O(\delta)$ accuracy. As expected on physical ground, the acoustic waves $\Delta p_{a, \xi}$ emitted by enthalpy perturbations act on the flame. However, enthalpy disturbances also modulate the flame stability directly via the factor $I_{0}$ in (6.8); this latter effect cannot be accounted for by a simple physical argument. This parametric instability thus differs from that caused by externally imposed acoustic waves, and may occur only when enthalpy fluctuations are present since (6.8) reduces to the familiar equation governing the D-L instability if $h_{-\infty}=0$. 
In the non-resonant case, it is necessary that $h_{-\infty}=O(1)$, and

$$
\Delta p_{a, \xi}(t)=\sum_{n=1}^{\infty} \frac{\left(\mathrm{i} \omega R_{+}\right) n j_{n}}{\Delta_{s}(n \omega ; \sigma)}\left\{1-\left(\frac{R_{-}}{R_{+}}\right)^{\frac{1}{2}} \tan \left(R_{-}^{\frac{1}{2}} \sigma n \omega L\right) \tan \left(R_{+}^{\frac{1}{2}}(1-\sigma) n \omega L\right)\right\} \mathrm{e}^{\mathrm{i} n \omega t}+c . c .
$$

For the resonant case, $h_{-\infty}=O\left(M^{\frac{1}{2}}\right)$, and consequently (6.8) simplifies to

$$
\left(R_{+}+R_{-}\right) \alpha^{\prime \prime}(t)+2 k \alpha^{\prime}(t)-\left\{q k^{2}+k\left(\left(R_{+}-R_{-}\right) G-\Delta p_{a, \xi}(t)\right)\right\} \alpha=0,
$$

where

$$
\Delta p_{a, \xi}(t)=2 \omega\left(1-R_{+} / R_{-}\right) R_{-}^{\frac{1}{2}} \sin \left(R_{-}^{\frac{1}{2}} \sigma \omega L\right) B(\tau) \mathrm{e}^{\mathrm{i} \omega t}+c . c . \equiv-\left(\chi_{c} B(\tau) \mathrm{e}^{\mathrm{i} \omega t}+c . c .\right),
$$

with $B$ being given by (5.15). Now $\Delta p_{a, \xi}$ varies with the slow time $\tau$ as well as with $t$, but the dependence on $\tau$ may be treated as being parametric, since the parametric instability occurs over a much shorter time scale.

According to Floquet theory, equation (6.8), or (A.16), admits solutions of the form

$$
\alpha=\mathrm{e}^{\mu t} \widehat{\alpha}(t)
$$

where $\widehat{\alpha}(t)$ is a periodic (and hence bounded) function of $t$ with period $2 \pi / \omega$, and $\mu$ is the Floquet exponent with its real part $\mu_{r}$ indicating stability (instability) according to $\mu_{r}<0\left(\mu_{r}>0\right)$. Solutions are found numerically by integrating (6.8) and (A.16) (with $\Delta p_{a}$ given by (6.9)) using a 4-th order Runge-Kutta method from $t=0$ to $t=2 \pi / \omega$ to obtain the principal fundamental matrix, from which $\mu$ can be calculated.

We first consider the case $U_{L}=10 \mathrm{cms}^{-1}$, and the gravity force is dropped (i.e. $G=0$ ). The enthalpy fluctuation is assigned a moderate amplitude $\hat{h}=0.6$, which corresponds to about $1 \%$ mass fraction fluctuation. The growth rates for different frequencies are shown in Fig.6a. An enthalpy fluctuation of moderate amplitude modifies the usual D-L instability (the dashed line): for most forcing frequencies the growth rate of the most unstable mode is enhanced, whilst the bandwidth of unstable modes becomes narrower. Of interest is the question whether an enthalpy fluctuation may have a net stabilizing effect, i.e. it reduces the unstable bandwidth without destabilizing the most unstable mode. A search indicates that that is realised only for an enthalpy perturbation with a frequency in a very small vicinity of the 'optimal' value $\omega=250$ (curve 1 ), which is about a $1 / 3$ of the frequency of the fundamental duct mode. An enthalpy disturbance with a frequency slightly above or below this is found to have a two-fold effect on the stability.

For a frequency fixed at the 'optimal' value $\omega=250$, the effect of increasing the enthalpy amplitude is displayed in fig.6b. As $\hat{h}$ increases to 0.7 , the maximum growth rate is reduced and the instability band is narrowed farther. However, as $\hat{h}$ is increased to 0.75, a secondary instability band consisting of larger transverse wavenumbers emerges. By monitoring Floquet multipliers, the onset of this band is found to be associated with the subharmonic parametric instability. When $\hat{h}$ reaches 0.8 , the second band becomes the dominant instability. Further calculations with $G \neq 0$ indicate that the subharmonic parametric instability also prevails when $\hat{h}$ exceeds a critical value, and it may completely destabilize a flame which is otherwise free of the D-L instability, that is, the parametric instability is a mechanism distinct from that of the D-L.

The result for the case $U_{L}=20 \mathrm{cms}^{-1}$ is shown in Fig.7. Now a moderate level of enthalpy disturbance fixed at $\hat{h}=0.6$ exerts a destabilising effect for all frequencies 

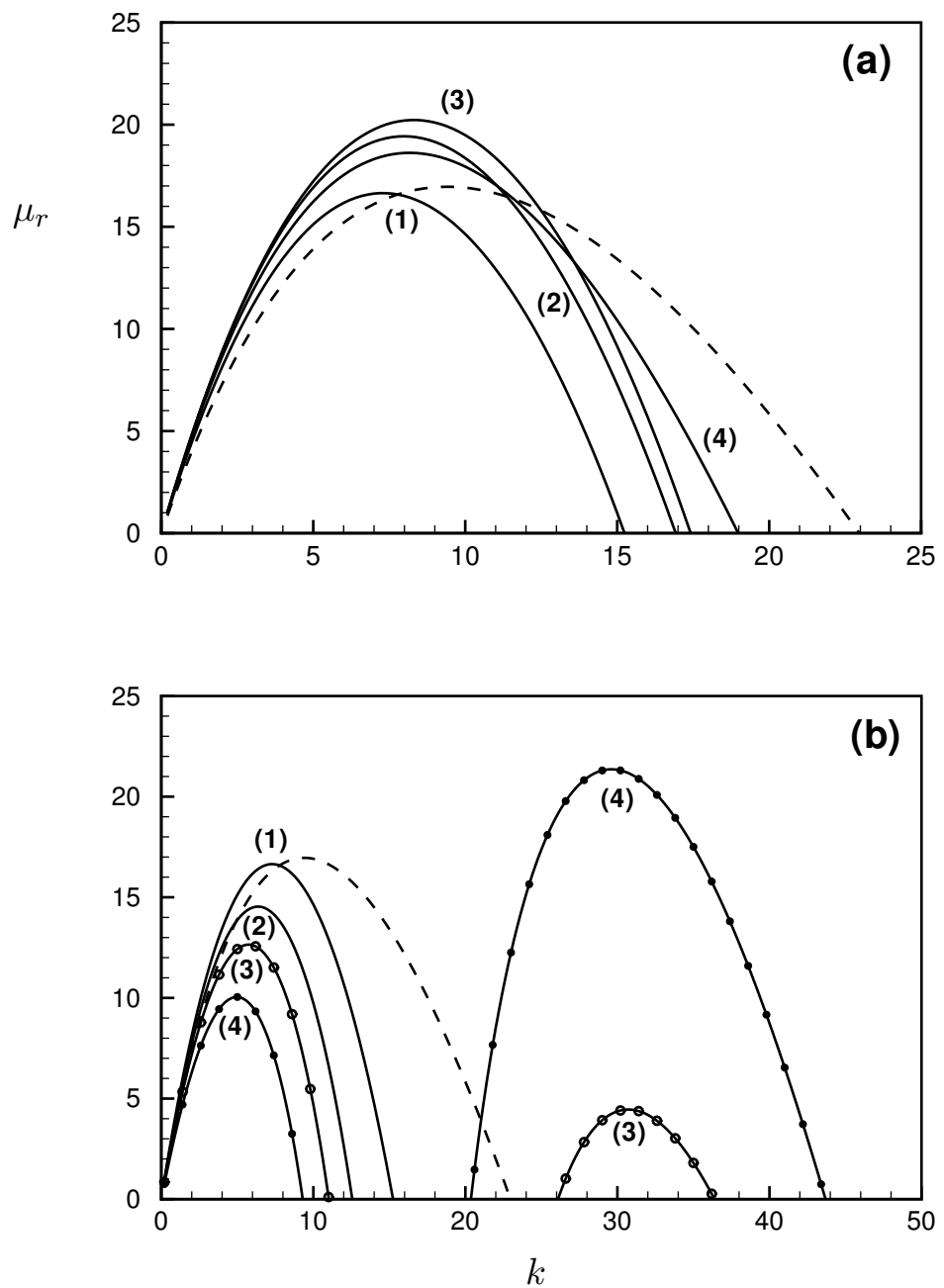

Figure 6: Parametric instability of the oscillatory planar flame with $U_{L}=0.1 \mathrm{~ms}^{-1}$. (a) Growth rates $\mu_{r}$ v.s. $k$ for different frequencies $\omega=250$ (curve 1), 300 (curve 2), 350 (curve 3 ) and $\omega=150$ (curve 4); the enthalpy disturbance amplitude $\hat{h}=0.6$. (b) Growth rates $\mu_{r}$ v.s. $k$ for different amplitudes $\hat{h}=0.6$ (curve 1), 0.7 (curve 2), 0.75 (curve 3 ) and 0.8 (curve 4); the enthalpy disturbance frequency $\omega=250$. The dashed line in each figure represents the D-L instability in the limit $\hat{h}=0$. 

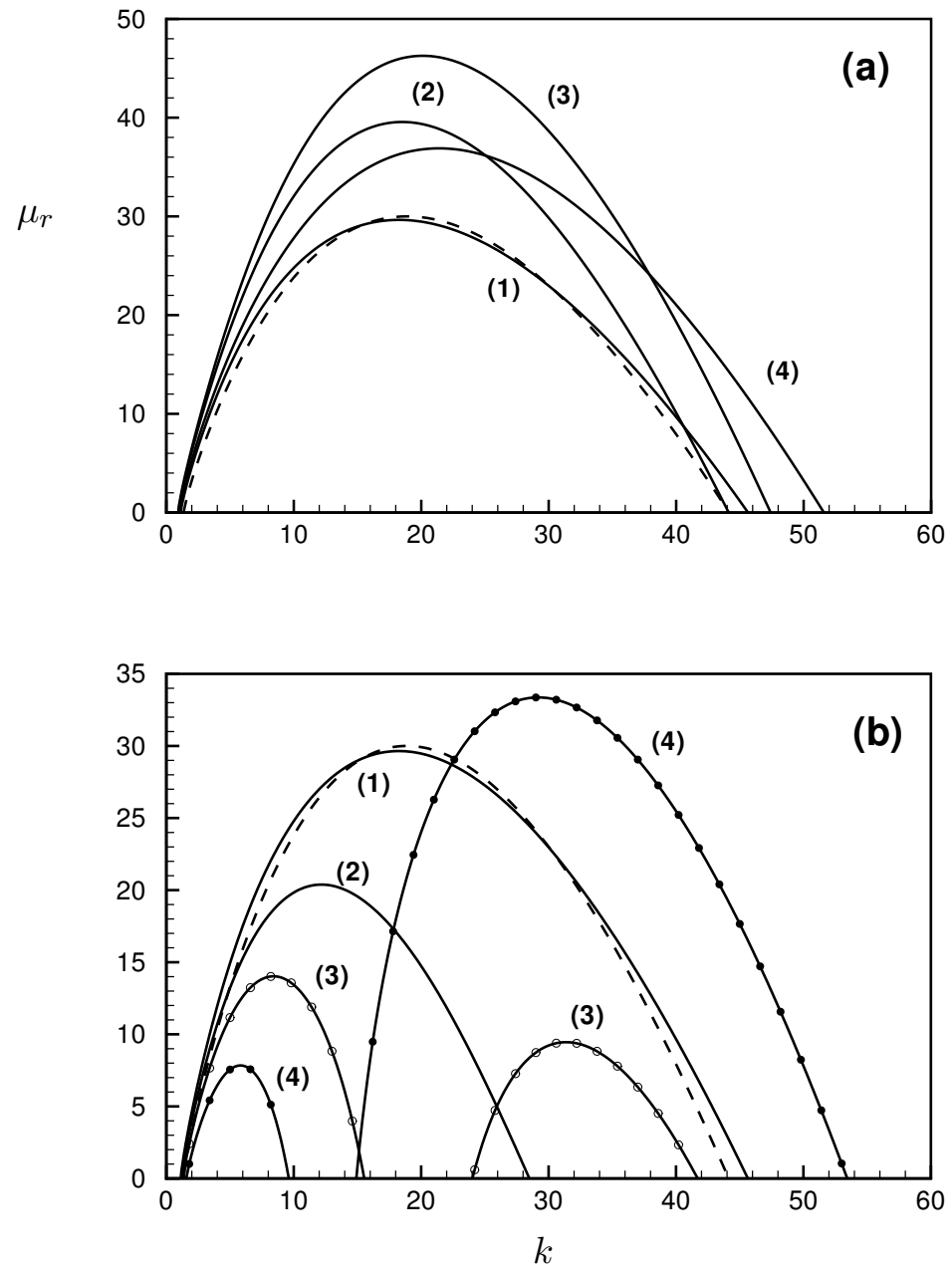

Figure 7: Parametric instability of the oscillatory planar flame with $U_{L}=0.2 \mathrm{~ms}^{-1}$. (a) Growth rates $\mu_{r}$ v.s. $k$ for different frequencies $\omega=125$ (curve 1), 225 (curve 2), 305 (curve 3) and 75 (curve 4). The enthalpy disturbance amplitude $\hat{h}=0.6$. (b) Growth rates $\mu_{r}$ v.s. $k$ for different amplitudes $\hat{h}=0.6$ (curve 1), 0.7 (curve 2), 0.75 (curve 3 ) and 0.8 (curve 4); the enthalpy disturbance frequency $\omega=125$. The dashed line in each figure represents the D-L instability in the limit $\hat{h}=0$. 


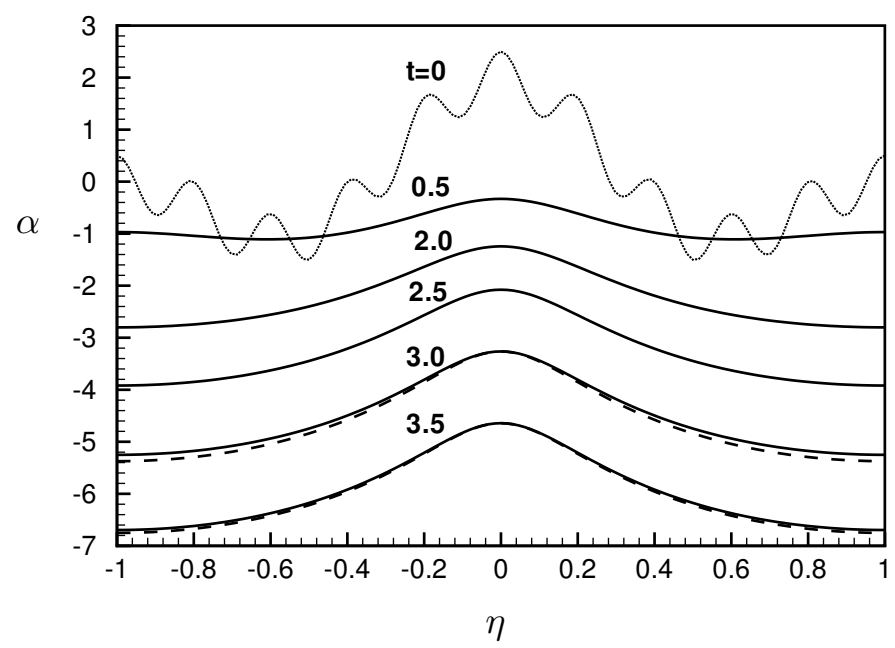

Figure 8: Solution to (7.3) when $\hat{h}=0$ as shown by flame front profiles at different times. The dashed lines represent the steady 1-pole solution, and dotted line represents the initial condition.

in that it increases the maximum growth rate while causing the unstable bandwidth to broaden (Fig.7a). The optimal frequency appears to be the one for which the growth-rate curve (curve 1) overlaps that of the D-L instability. Increasing the enthalpy amplitude has the same effect as observed in the case of $U_{L}=10 \mathrm{cms}^{-1}$. The D-L modes in the lowwavenumber band are stabilized, but a second band of subharmonic parametric instability emerges and soon becomes dominant (Fig.7b).

Fig.6b and Fig.7b both suggest that at a fixed $\omega$, there may exist a small interval of $\hat{h}$ (between 0.6 and 0.7 ) for which the enthalpy has a stabilizing effect. Such an interval may be continued to identify a stabilizing region in the $\hat{h}-\omega$ parameter plane. Such a delicate search has not been pursued here, because it is better to be performed for parameters pertaining to a particular experimental condition.

\section{$7 \quad$ Nonlinear instability in the limit of small heat release}

For the problems considered in $\S \S 4-6$, the flame is governed by linear dynamics. In this section, we study weakly nonlinear development of a flame in the limit of small heat release, $q \ll 1$. The focus will be on the non-resonant case, which is of interest as experiments indicate that modulating the fuel at low frequencies (about $1 / 4$ the fundamental acoustic frequency of the combustor or even lower) is effective in stabilizing combustion (e.g. Jones et al. 1999, Richards et al. 1999).

Suppose that $F=O(\epsilon)$. The jump conditions imply that $U, \mathbf{V}, P \sim O(q \epsilon)$. In the absence of enthalpy fluctuations, the gas expansion effect drives a weak hydrodynamic instability with an $O(q)$ growth rate, as can be inferred from (6.8). Then the hydrodynamic field is governed by linear quasi-steady equations to the first order of approximation. The geometric nonlinearity in the front equation (3.22) is of $O\left(\epsilon^{2}\right)$, comparable with $U^{-}$and must be retained when $\epsilon=O(q)$. In this case, the well-known Michelson-Sivashinsky (M-S) equation can be derived (Sivashinsky 1977). This equation has been extended/modified in 


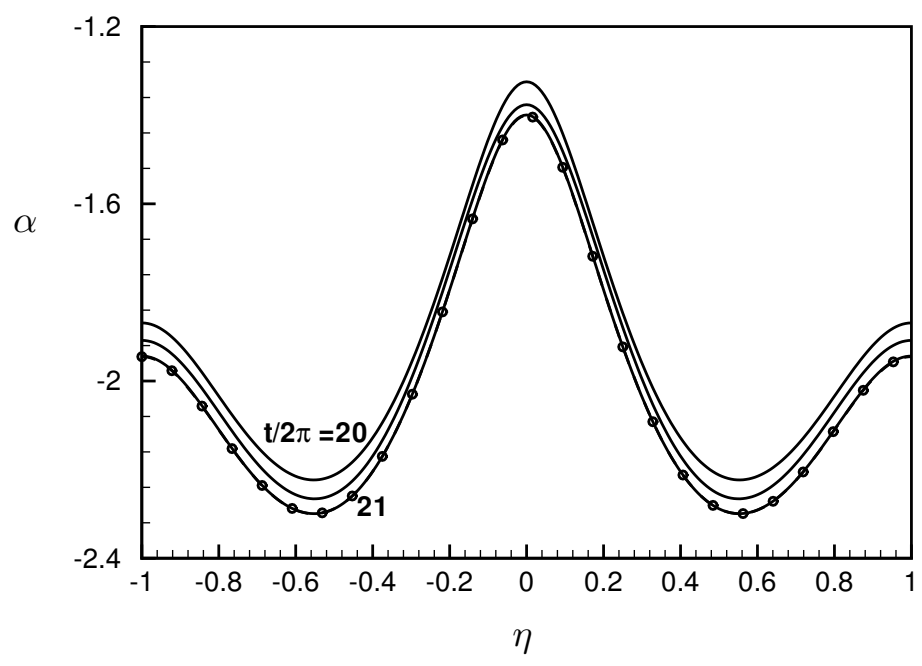

Figure 9: Solution to (7.3) when $\hat{h}=0.6$ and $\nu=0.44$ as shown by flame front profiles at different times.

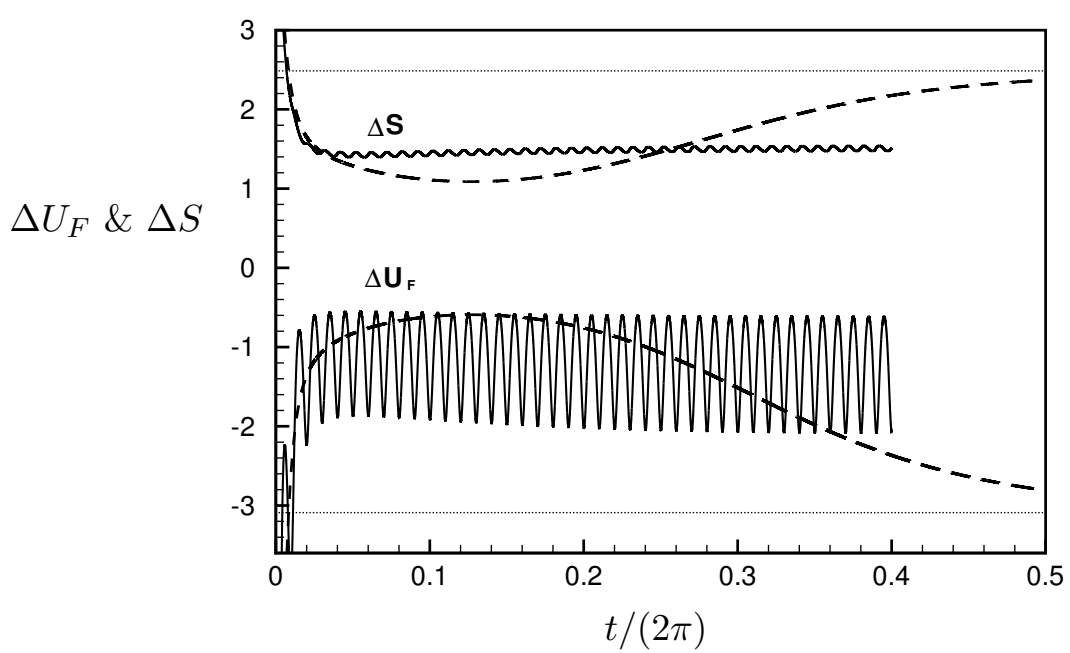

Figure 10: Flames speed $\Delta U_{F}$ and surface area alteration $\Delta S$ v.s. time $t$ for $\nu=0.44$ and $\hat{h}=0.6$ (solid lines), and the comparison with the corresponding result for $\hat{h}=0$ (dashed lines). 


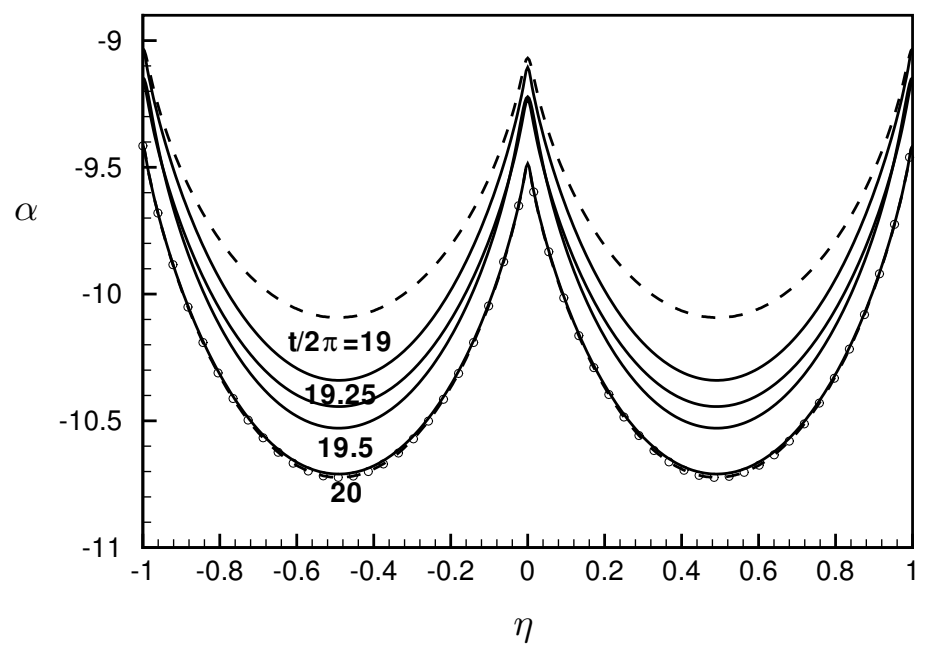

Figure 11: Solution to (7.3) when $\hat{h}=0.6$ and $\nu=0.044$ as shown by flame front profiles at different times. The dashed line represents the steady solution attained if $\hat{h}=0$.

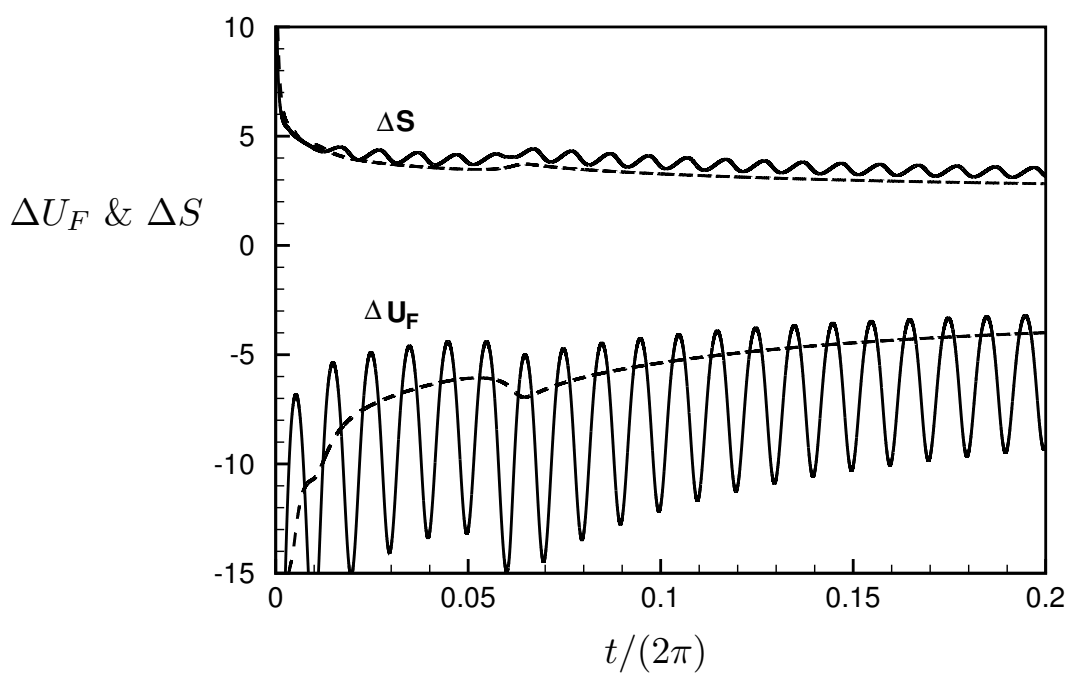

Figure 12: Flames speed $\Delta U_{F}$ and surface area alteration $\Delta S$ v.s. time $t$ for $\nu=0.044$ and $\hat{h}=0.6$ (solid lines), and the comparison with the corresponding result for $\hat{h}=0$ (dashed lines). 
various ways and applied to flames with $q=O(1)$ (e.g. Joulin \& Cambray 1992). However, equations of this type have two limitations: (a) they cannot describe short-time transient behaviours of a flame, and (b) the acoustic field generated by the flame is ignored.

When an enthalpy disturbance $h_{-\infty}(t)$ is present, acoustic acceleration $\Delta p_{a, \xi} \sim O(q)$ is induced. The flow evolves over two time scales: a short scale $t \sim O(1)$ associated with $h_{-\infty}(t)$ and $\Delta p_{a, \xi}$, and a much longer intrinsic scale $t \sim O\left(q^{-1}\right)$, over which the instability develops. For this reason, the fully unsteady but linearised hydrodynamic equations will be employed, while the front equation takes, to $O\left(\epsilon^{2}\right)$ accuracy, the nonlinear form

$$
F_{t}=U_{0}\left(0^{-}, t\right)-\frac{1}{2} \mathrm{e}^{\frac{1}{2} h_{-\infty}}|\nabla F|^{2}+\nu \nabla^{2} F,
$$

where $\nu=\delta M_{a}$. Here it has been further assumed that $\delta=O(\epsilon)$ in order to retain the effect of flame curvature. This term renders the initial-value problem well-posed as it sets a high wavenumber cut-off beyond which the D-L instability is stabilized.

In Fourier spectral space, the solution to (6.2) is given by (6.3) with (6.4)-(6.5). By eliminating $C_{+}, \phi_{+}$and $\mathbf{D}_{+}$from (6.6) and (6.7), it can be shown that

$$
\phi_{-}^{\prime}+\frac{2 k \mathrm{e}^{\frac{1}{2} h_{-\infty}}}{R_{+}+R_{-}} \phi_{-}=\frac{k\left\{\left(R_{+}-R_{-}\right) G-\Delta p_{a, \tilde{\xi}}\right\}+q k^{2} \mathrm{e}^{h_{-\infty}}}{R_{+}+R_{-}} \alpha .
$$

The equation can easily be solved to find $\phi_{-}$, which is inverted to obtain $U$ and then substituted into (7.1) to give

$$
F_{t}+\frac{1}{2} \mathrm{e}^{\frac{1}{2} h_{-\infty}(t)}|\nabla F|^{2}=\nu \nabla^{2} F+\mathcal{I}
$$

where

$$
\begin{aligned}
\mathcal{I}=\frac{1}{4 \pi^{2}} \int_{0}^{t}\{ & \left.\int_{-\infty}^{\infty} \int_{-\infty}^{\infty}\left|\mathbf{k}^{\dagger}\right| F(t-\tau, \tilde{\eta}) K(k, t, \tau) \mathrm{e}^{\mathrm{i} \mathbf{k}^{\dagger} \cdot(\eta-\tilde{\eta})} \mathrm{d} \tilde{\eta} \mathrm{d} \mathbf{k}^{\dagger}\right\} \mathrm{d} \tau \\
K(k, t, \tau)= & \frac{1}{R_{+}+R_{-}}\left\{\left(R_{+}-R_{-}\right) G-\Delta p_{a, \tilde{\xi}}(t-\tau)+q k \mathrm{e}^{h_{-\infty}(t-\tau)}\right\} \\
& \times \exp \left\{-\frac{2 k}{R_{+}+R_{-}} \int_{0}^{\tau} \mathrm{e}^{\frac{1}{2} h_{-\infty}(t-\tilde{\tau})} \mathrm{d} \tilde{\tau}\right\} .
\end{aligned}
$$

It is noted that treating the hydrodynamic field as being fully unsteady leads to historydependent integro-differential equation.

When enthalpy disturbances and gravity are both absent, equation (7.3) reduces to

$$
F_{t}+\frac{1}{2}|\nabla F|^{2}=\nu \nabla^{2} F+\mathcal{I}
$$

where

$$
\mathcal{I}=\frac{q}{4 \pi^{2}\left(R_{+}+R_{-}\right)} \int_{0}^{t}\left\{\int_{-\infty}^{\infty} \int_{-\infty}^{\infty} k^{2} F(t-\tau, \tilde{\eta}) \exp \left\{-\frac{2 k \tau}{R_{+}+R_{-}}\right\} \mathrm{e}^{\mathrm{i} \mathbf{k}^{\dagger} \cdot(\eta-\tilde{\eta})} \mathrm{d} \tilde{\eta} \mathrm{d} \mathbf{k}^{\dagger}\right\} \mathrm{d} \tau .
$$

Now without acoustic and enthalpy fluctuations, there is no natural fast time scale. The conventional approximation of neglecting the time-derivative term in (7.2) leads to the familiar M-S equation (Sivashinsky 1977)

$$
F_{t}+\frac{1}{2}|\nabla F|^{2}=\nu \nabla^{2} F+\frac{q}{8 \pi^{2}} \int_{-\infty}^{\infty} \int_{-\infty}^{\infty}\left|\mathbf{k}^{\dagger}\right| F(t, \tilde{\eta}) \mathrm{e}^{\mathrm{i} \mathbf{k}^{\dagger} \cdot(\eta-\tilde{\eta})} \mathrm{d} \tilde{\eta} \mathrm{d} \mathbf{k}^{\dagger}
$$


which is somewhat simpler than (7.5) in that it is local in time. However, the approximation employed implies that (7.6) is not applicable to the transient phase occurring on the time scale $t \sim O(1)$, whilst (7.5) (which may be called non-local M-S equation) has the advantage that it describes the entire evolution from a general initial condition. Matalon \& Metzener (1997) found that taking into account this transient effect is crucial for explaining the formation of the so-called 'tulip flame' in a closed tube.

In the limit $q \ll 1$, equation (7.3) stands as a self-consistent approximate equation (with $O(\epsilon)$ accuracy) that governs the nonlinear development of broad-band instability waves. Unfortunately, the self-consistency is lost for $q=O(1)$. Nevertheless, a noticeable feature of (7.3) is that the linear instability is exactly the same as for the general case with $q=O(1)$, that is, it immediately reduces to system (6.8) if the geometric nonlinear term is ignored. Therefore even when $q=O(1)$, equation (7.3) is expected to have some validity provided that nonlinearity is not too strong.

In the general case, the evolution equation (7.3) in physical space is rather complex, and may not be best suited for numerical studies. It is more convenient to work in Fourier space instead. The nonlinear front equation is Fourier transformed, and the resulting equation is combined with (7.2) to form the system

$$
\left.\begin{array}{c}
\alpha^{\prime}=-\nu k^{2} \alpha+\phi_{-}+\frac{1}{2} \mathrm{e}^{\frac{1}{2} h_{-\infty}} \int_{-\infty}^{\infty} \tilde{\mathbf{k}}^{\dagger} \cdot\left(\mathbf{k}^{\dagger}-\tilde{\mathbf{k}}^{\dagger}\right) \alpha\left(\mathbf{k}^{\dagger}-\tilde{\mathbf{k}}^{\dagger}\right) \alpha\left(\tilde{\mathbf{k}}^{\dagger}\right) \mathrm{d} \tilde{\mathbf{k}}^{\dagger}, \\
\phi_{-}^{\prime}+\frac{2 k \mathrm{e}^{\frac{1}{2} h_{-\infty}}}{R_{+}+R_{-}} \phi_{-}=\frac{k\left\{\left(R_{+}-R_{-}\right) G-\Delta p_{a, \tilde{\xi}}\right\}+q k^{2} \mathrm{e}^{h_{-\infty}}}{R_{+}+R_{-}} \alpha .
\end{array}\right\}
$$

For a two-dimensional flame in a finite domain $-1 \leq \eta \leq 1$, the solution for $F$ may be expressed as

$$
F(\eta, t)=\sum_{n=-\infty}^{\infty} f_{n}(t) \mathrm{e}^{n \mathrm{i} \pi \eta} .
$$

A discrete version of (7.7) holds for $f_{n} \equiv \alpha(k)$ and $\phi_{n} \equiv \phi_{-}(k)$ with $k= \pm n \pi(n=$ $0,1,2, \ldots)$. This nonlinear system is solved numerically by using a pseudo-spectral method.

As a validation of the computational code, the non-local M-S equation in the limit $\hat{h}=0$ is solved first. The front profiles at different times are displayed in Fig.8. Consistent with previous findings (e.g. Rastigejev \& Matalon 2006), the solution approaches a steady state, which for $\nu=0.44$ corresponds to a coalescence 1-pole solution (Rahibe, Aubry \& Sivashinsky 1996, Vaynblat \& Matalon 1999). The numerical solution is in good agreement with the exact solution.

In the presence of an enthalpy disturbance with an amplitude $\hat{h}=0.6$, the solution evolves into a periodic state. The profiles at three instants over one period is shown in Fig.9. The overall shape is very different from that of the steady solution attained when $\hat{h}=0$. On the other hand, the shape of the flame front hardly changes with time.

Of particular interest are the averaged flame speed $\Delta U_{F}$ and the alteration of the flame surface area $\Delta S$, which may be defined respectively as

$$
\Delta U_{F}=\frac{\mathrm{d}}{\mathrm{d} t} \bar{F}=f_{0}^{\prime}(t), \quad \Delta S=\left[\overline{|\nabla F|^{2}}\right]^{1 / 2}=\left[\sum_{n=1}(n \pi)^{2}\left|f_{n}(t)\right|^{2}\right]^{1 / 2} .
$$

They are used to characterize the overall flame response to enthalpy fluctuations. The evolution of $\Delta S$ and $\Delta U_{F}$ for $\nu=0.44$ is displayed in Fig.10. The surface area is modulated periodically about its mean value, but the oscillation magnitude is very small, consistent 
with the profile shown in Fig.9. In contrast, the overall flame speed oscillates at a large amplitude, suggesting that the flame front vibrates almost rigidly in the longitudinal direction. It is interesting to note that despite the relatively moderate amplitude of the enthalpy perturbation, both the mean surface area and mean flame speed differ appreciably from those when enthalpy fluctuation is absent.

We also solved the extended M-S equation for a small $\nu=0.044$. Again the solution approaches a periodic state (Fig.11). The flame would appear to be breathing periodically without significantly changing its overall shape, which is similar to the steady solution attained when $\hat{h}=0$, but is more elongated. As is illustrated by Fig.12, the surface area varies slightly about its mean value, but there is a significant modulation in the overall flame speed, the mean value of which is about $50 \%$ higher than that for $\hat{h}=0$.

\section{Discussion and conclusions}

Instability of premixed combustion involves intricately coupled physical processes, which occur over a vast range of length scales. While presenting a great challenge for DNS, the multi-scale nature of the problem can be exploited mathematically in AEA. This framework has been employed to derive simplified theories describing flame-flow interactions. The theory pertinent to the 'corrugate flamelet' regime was given by Matalon \& Matkowsky (1982) and Pelce \& Clavin (1982). Based on their theory, a general formulation for flame-acoustic coupling has been developed recently (WWMP).

In most existing AEA theories of premixed flames, the fuel mass fraction and temperature of the oncoming mixture are both assumed to be steady in time and uniform in space, or when variations are represent the constant-density assumption is made. In the present paper, these restrictive assumptions were removed, and an asymptotic theory for premixed combustion under the influence of enthalpy fluctuations was derived from the reactive N-S equations. Analogous to the well-known work of Matalon \& Matkowsky (1982) and Pelce \& Clavin (1982), the flame is represented by a single order parameter, the front position $F$, and its interaction with the ambient flow motion is described by velocity and pressure jumps. By analysing the preheat zone, these jumps are obtained to the second-order accuracy, and are found to be augmented by $h_{-\infty}$, the local enthalpy fluctuation advected to the flame front. The generation of spontaneous sound waves by the flame and their back action were analysed leading to a fully interactive system that describes the flame-acoustic-enthalpy coupling; see figure 1.

The general asymptotic theory was applied to several special cases, shedding useful light on the role of enthalpy fluctuations in premixed combustion. The calculation of the linear response of a planar flame to small-amplitude enthalpy perturbations ( $\$ 4)$ indicates that spatially non-uniform enthalpy fluctuations directly influence the hydrodynamic motion. The analysis for one-dimensional enthalpy fluctuations $(\S 5)$ highlights the fact that by modulating the heat release, unsteady enthalpy perturbations affect the generation of acoustic waves, or even emit sound waves directly. The linear stability analysis $(\S 6)$ reveals that enthalpy fluctuations modify the D-L instability, and may induce much more vigorous subharmonic parametric instability. Finally, possible impact of enthalpy on the weakly nonlinear flame dynamics was demonstrated by the extended M-S equation, derived and studied in $\S 7$. It may be noted that none of the effects highlighted here could be accounted for by a thermal-diffusive model or the G-equation approach.

The present paper represents only a first step towards modelling the influence of enthalpy disturbances on premixed combustion on the basis of first principles. Several de- 
velopments may be followed. A thorough investigation of the fully nonlinear system is yet to be undertaken. Compared with the full reactive Euler or N-S equations, the asymptotically reduced system gives us the computational advantage that chemical reactions, gas expansion and acoustics are accounted for without the need of resolving numerically the thin flame zone or solving the full compressible equations. However, the front equation has to be solved in conjunction with the Euler (or N-S) equations, and so accurate front tracking or capturing algorithms are required.

The present asymptotic theory was derived for the 'open loop' case. It would be interesting to extend it to 'closed loop' situations, so that one may study the mechanism (d) of combustion instability. A further ingredient to be added is a suitable model describing how the feeding line responds to the acoustic signature. Such a physics-based theory would have important applications to active control using secondary fuel injection.

Combustion in most practical applications takes place in turbulent flows. Since it will remain infeasible in a foreseeable future for DNS to resolve thin flame zones or reaction sheets, the main prediction tools will be the Reynolds-averaged equations or large-eddy simulations. Therefore, in addition to modelling sub-scale/grid turbulence, it is necessary to model flame-turbulence interaction, the most important among which concerns modelling the turbulent flame speed (Peters 2000, Pitsch 2005). Theoretical understandings gained from AEA analyses for laminar flames have, through the flamelet concept, aided the modelling effort (Peters 2000). However, almost all models ignore the effects of the spontaneously emitted acoustic waves and the oncoming enthalpy perturbations. It would be interesting to develop improved models to include these effects by exploiting the asymptotic formulations. Since enthalpy fluctuations influence the flamelet motion, the turbulent flame speed $S_{T}$ would depend on $\overline{h^{\prime 2}}$ as well as on $\overline{\mathbf{u}^{\prime 2}}$, the rms of the velocity fluctuations. Furthermore, considering that the flame dynamics is fundamentally influenced by the gas expansion and thermal-diffusive effects, which are characterised by $q$ and Le respectively, one may postulate that $S_{T}$ should be parameterised as $S_{T}=S_{T}\left(\overline{\mathbf{u}^{\prime 2}}, \overline{h^{\prime 2}} ; q\right.$, Le).

The work of XW was carried out during his sabbatical leave (May-October 2008) in the Center of Turbulence Research, Stanford University.

\section{A Second-order theory for the parametric instability}

It follows from (3.22) that the position of the planar flame is, to $O(\delta)$ accuracy, given by

$$
F(t)=\int^{t}\left(1-I^{-}(t)\right) \mathrm{d} t
$$

where

$$
I^{-}(t)=I_{0}+\delta\left[M_{a}+\ln (1+q)+q /(1+q)\right] I_{0}^{\prime} / I_{0}^{2} .
$$

The acoustic source associated with the flame is given by

$$
\mathcal{J}=\left[\left[u_{a}\right]\right]=q\left(I_{0}-1\right)+\delta q\left[\ln (1+q)+q /(1+q)+\frac{1}{2} l \mathcal{D}\right] I_{0}^{\prime} / I_{0}^{2} .
$$

This is used to calculate $\Delta p_{a, \tilde{\xi}}$, which acts on the flame.

The planar flame is perturbed by a small-amplitude disturbance $\tilde{F} \ll 1$. The hydro- 
dynamic motion $(\tilde{U}, \tilde{\mathbf{V}}, \tilde{P}) \ll 1$ is governed by the linearised version of (3.8), i.e.,

$$
\left.\begin{array}{c}
\frac{\partial \tilde{U}}{\partial \xi}+\nabla \cdot \tilde{\mathbf{V}}=0 \\
\left\{R_{+} \frac{\partial}{\partial t}+I^{+}(t) \frac{\partial}{\partial \xi}-\delta \operatorname{Pr}\left(\frac{\partial^{2}}{\partial \xi^{2}}-k^{2}\right)\right\}\left(\begin{array}{c}
U \\
\mathbf{V}
\end{array}\right)=-\left(\begin{array}{l}
\partial / \partial \xi \\
\nabla
\end{array}\right) \tilde{P}
\end{array}\right\}
$$

where

$$
I^{+}=I^{-}-\delta \ln (1+q) I_{0}^{\prime} / I_{0}^{2}=I_{0}+\delta\left[M_{a}+q /(1+q)\right] I_{0}^{\prime} / I_{0}^{2} .
$$

With the viscous diffusion terms being included in the hydrodynamic equations, the solution for $(\tilde{U}, \tilde{\mathbf{V}}, \tilde{P})$ can still be written as (6.3) with (6.4)-(6.5), but the functions $C_{+}$and $\mathbf{D}_{+}$must be replaced by $C^{+}(\xi, t)$ and $\mathbf{D}^{+}(\xi, t)$, and the function $I_{0}$ in $(6.5)$ replaced by $I^{ \pm}$for $\xi>0$ and $\xi<0$ respectively. Functions $C^{+}(\xi, t)$ and $\mathbf{D}^{+}(\xi, t)$ now satisfy

$$
\begin{gathered}
C_{\xi}^{+}+\mathrm{i} \mathbf{k}^{\dagger} \cdot \mathbf{D}^{+}=0 \\
\left\{R_{+} \frac{\partial}{\partial t}+I^{+}(t) \frac{\partial}{\partial \xi}-\delta \operatorname{Pr}\left(\frac{\partial^{2}}{\partial \xi^{2}}-k^{2}\right)\right\}\left(\begin{array}{l}
C^{+} \\
\mathbf{D}^{+}
\end{array}\right)=0 .
\end{gathered}
$$

Let $\widehat{C}^{+}(s, t)$ and $\widehat{\mathbf{D}}^{+}(s, t)$ denote respectively the Laplace transforms of $C^{+}$and $\mathbf{D}^{+}(\xi, t)$ with respect to $\xi>0$. Equations (A.6) are solved to give

$$
\begin{aligned}
\widehat{C}^{+}(s, t)= & -\frac{R_{+}^{-1}}{s \Lambda} \int_{-\infty}^{t}\left\{R_{+} C_{\tilde{t}}^{+}\left(0^{+}, \tilde{t}\right)+\delta \operatorname{Pr} k^{2} C^{+}\left(0^{+}, \tilde{t}\right)+\delta \operatorname{Pr} s C_{\xi}^{+}\left(0^{+}, \tilde{t}\right)\right\} \Lambda(\tilde{t}) \mathrm{d} \tilde{t} \\
& +s^{-1} C^{+}\left(0^{+}, t\right), \\
\widehat{\mathbf{D}}^{+}(s, t)= & \frac{R_{+}^{-1}}{\Lambda(t)} \int_{-\infty}^{t}\left\{\left[I^{+}(\tilde{t})-\delta \operatorname{Pr} s\right] \mathbf{D}^{+}\left(0^{+}, \tilde{t}\right)-\delta \operatorname{PrD}_{\xi}^{+}\left(0^{+}, \tilde{t}\right)\right\} \Lambda(\tilde{t}) \mathrm{d} \tilde{t},
\end{aligned}
$$

where

$$
\Lambda(t)=\exp \left\{R_{+}^{-1} \int^{t}\left[s I^{+}(\tau)-\delta \operatorname{Pr}\left(s^{2}-k^{2}\right)\right] \mathrm{d} \tau\right\} .
$$

The continuity equation (A.5) is Laplace transformed to

$$
s \widehat{C}^{+}(s, t)-C^{+}(0, t)+\mathrm{i} \mathbf{k}^{\dagger} \cdot \widehat{\mathbf{D}}^{+}=0 .
$$

Inserting (A.7) and (A.8) into (A.9) and making use of (A.5), we obtain the relation

$$
-R_{+} C_{t}^{+}\left(0^{+}, t\right)-\delta \operatorname{Pr} k^{2} C^{+}\left(0^{+}, t\right)+I^{+} \mathrm{i} \mathbf{k}^{\dagger} \cdot \mathbf{D}^{+}\left(0^{+}, t\right)-\delta \operatorname{Pr} \mathbf{i} \mathbf{k}^{\dagger} \cdot \mathbf{D}_{\xi}^{+}\left(0^{+}, t\right)=0 .
$$

Use of the solution (6.4) in the velocity jump conditions (3.12) and (3.15) shows that

$$
\begin{aligned}
C^{+}\left(0^{+}, t\right) & =-\left(\phi_{+}-\phi_{-}\right)+\delta\left(\frac{1}{2} l q \mathcal{D}\right)\left(k^{2} \alpha+k \phi_{-} I_{0}\right) \\
\mathbf{D}^{+}\left(0^{+}, t\right)= & \frac{\mathrm{i} \mathbf{k}^{\dagger}}{k}\left(\phi_{+}+\phi_{-}\right)-q I\left(\mathrm{i} \mathbf{k}^{\dagger}\right) \alpha+\delta\left\{\frac{\operatorname{Pr}}{I_{0}}\left[\mathbf{D}_{\xi}^{+}\left(0^{+}, t\right)+\mathrm{i} \mathbf{k}^{\dagger}\left(\phi_{+}-\phi_{-}\right)\right]\right. \\
& \left.+\frac{\mathrm{i} \mathbf{k}^{\dagger}}{I_{0}^{2}}\left[\frac{1}{k} \phi_{-}^{\prime}(t)+I_{0} \alpha^{\prime}+\left(G+u_{a, t}\left(0^{-}, t\right)\right) \alpha\right] \ln (1+q)\right\}
\end{aligned}
$$


where $I=I_{0}+\delta\left[\ln (1+q)+q /(1+q)+\frac{1}{2} l \mathcal{D}\right] I_{0}^{\prime} / I_{0}^{2}$, as can be found from (6.4). The above relations are inserted into (A.10) to give

$$
\begin{aligned}
& R_{+}\left(\phi_{+}^{\prime}-\phi_{-}^{\prime}\right)-I^{+} k\left(\phi_{+}+\phi_{-}\right)+q I I^{+} k^{2} \alpha-\delta \frac{\ln (1+q)}{I_{0}}\left(G+u_{a, t}\left(0^{-}, t\right)\right) k^{2} \alpha \\
& \quad=\delta\left\{\left[\ln (1+q)+\frac{q l \mathcal{D}}{2(1+q)}\right]\left(k^{2} \alpha^{\prime}+\frac{k \phi_{-}^{\prime}}{I_{0}}\right)-\frac{q l \mathcal{D} I_{0}^{\prime}}{2(1+q) I_{0}^{2}} k \phi_{-}\right\} .
\end{aligned}
$$

On substituting the solution for $\tilde{P}$ into (3.16), the pressure jump can be written as

$$
\begin{gathered}
R_{+} \phi_{+}^{\prime}+R_{-} \phi_{-}^{\prime}-k\left(I^{+} \phi_{+}-I^{-} \phi_{-}\right)-k\left\{\left(R_{+}-R_{-}\right) G-\Delta p_{a, \xi}\right\} \alpha \\
=\delta k\left\{-q l \mathcal{D}\left(I_{0} k^{2} \alpha+k \phi_{-}\right)-q I_{0} k^{2} \alpha+\ln (1+q) \phi_{-}^{\prime} / I_{0}\right\} .
\end{gathered}
$$

Subtracting (A.13) from (A.14) leads to

$$
\begin{gathered}
\left(R_{+}+R_{-}\right) \phi_{-}^{\prime}+k\left(I^{+}+I^{-}\right) \phi_{-}-\left\{q I I^{+} k^{2}+k\left[\left(R_{+}-R_{-}\right) G-\Delta p_{a, \xi}\right]\right\} \alpha \\
=-\delta\left\{q l \mathcal{D}\left(I_{0} k^{3} \alpha+k^{2} \phi_{-}\right)+q I_{0} k^{3} \alpha+\left[\ln (1+q)+\frac{q l \mathcal{D}}{2(1+q)}\right] k^{2} \alpha^{\prime}\right. \\
\left.+\frac{q l \mathcal{D}}{2(1+q)} k\left[\frac{\phi_{-}^{\prime}}{I_{0}}-\frac{I_{0}^{\prime} \phi_{-}}{I_{0}^{2}}\right]+\frac{\ln (1+q)}{I_{0}}\left(G+u_{a, t}\left(0^{-}, t\right)\right) k^{2} \alpha\right\}
\end{gathered}
$$

The front equation (3.22) becomes $\alpha^{\prime}=\phi_{-}-\delta M_{a}\left(k^{2} \alpha+k \phi_{-} / I_{0}\right)$. Combining this equation with (A.15) to eliminate $\phi_{-}$in favour of $\alpha$, we obtain the equation

$$
\tilde{\mathcal{A}} \alpha^{\prime \prime}+\tilde{\mathcal{B}} \alpha^{\prime}+\tilde{\mathcal{C}} \alpha=0
$$

which governs the parametric instability, where $\tilde{\mathcal{A}}, \tilde{\mathcal{B}}$ and $\tilde{\mathcal{C}}$ are functions of $t$, given by

$$
\begin{aligned}
\tilde{\mathcal{A}}= & \left(R_{+}+R_{-}\right)+\delta I_{0}^{-1}\left\{\frac{q+2}{q} \ln (1+q)+l \mathcal{D}\right\} k, \\
\tilde{\mathcal{B}}= & 2 k I_{0}+\delta\left\{\left[\frac{4(q+1)}{q} \ln (1+q)+(q+2) l \mathcal{D}\right] k^{2}+2\left[\ln (1+q)+\frac{q}{1+q}\right] \frac{I_{0}^{\prime}}{I_{0}^{2}} k\right\}, \\
\tilde{\mathcal{C}}=- & \left\{q I_{0}^{2} k^{2}+k\left[\left(R_{+}-R_{-}\right) G-\Delta p_{a, \xi}\right]-\delta \frac{\ln (1+q)}{I_{0}}\left(G+u_{a, t}\left(0^{-}, t\right)\right) k^{2}\right\} \\
& +\delta\left\{\left[\frac{2(q+1)}{q} \ln (1+q)+(q+1) l \mathcal{D}+q\right] I_{0} k^{3}-q\left[\frac{1+2 q}{q} \ln (1+q)+\frac{q}{1+q}+\frac{1}{2} l \mathcal{D}\right] \frac{I_{0}^{\prime}}{I_{0}} k^{2}\right\} .
\end{aligned}
$$

When $h_{-\infty}=0, I=I_{0}=1$ so that $\tilde{\mathcal{A}}, \tilde{\mathcal{B}}$ and $\tilde{\mathcal{C}}$ simplify, and (A.16) reduces to the equation governing the D-L instability to $O(\delta)$ accuracy. 


\section{References}

[1] Aldredge, R. C. \& Williams, F. A. 1991 Influence of wrinkled premixed-flame dynamics on large scale, low-intensity turbulent flow. J. Fluid Mech. 228, 487-511.

[2] Baillot, F., Durox, D. \& Prud'homme, R. 1992 Experimental and theoretical study of a premixed vibrating flame. Combust. Flame, 88(2), 149-168.

[3] Birbaud, A.L., Ducruix, S., Durox, D. \& Candel, S. 2008 The nonlinear response of inverted "V" flames to equivalence ratio nonuniformities. Combust. Flame 154, 356-367.

[4] Candel, S. 2002 Combustion dynamics and control: progress and challenges. Proc. Comb. Inst. 29, 1-28.

[5] Cho, J.H. \& Lieuwen, T. 2005 Laminar premixed flame response to equivalence ratio oscillations. Combust. Flame 140, 116-129.

[6] Clavin, P. 1994 Premixed combustion and gasdynamics. Annu. Rev. Fluid Mech. 26, 321-352.

[7] Clavin, P. 2000 Dynamics of combustion fronts in premixed gases: from flames to detonations. Proc. Comb. Inst. 28, 569-585.

[8] Clavin, P., Pelce, P. \& He, L. 1990 One-dimensional vibratory instability of planar flames propagating in tubes. J. Fluid Mech. 216, 299-322.

[9] Dowling, A.P. \& Morgans, A.S. 2005 Feedback control of combustion oscillations. Annu. Rev. Fluid Mech. 37, 151-182.

[10] Daou J., Matalon, M. \& Linan, A. 2000 Premixed edge-flames under transverse enthalpy gradients. Combust. Flame 121, 107-121.

[11] Ducruix, S., Durox, D. \& Candel, S. 2000 Theoretical and experimental determination of the transfer function of laminar premixed flame. Proc. Comb. Inst. 28, 765-773.

[12] Ducruix, S., Schuller, T., Durox, D. \& Candel, S. 2003 Combustion dynamics and instabilities: elementary coupling and driving mechanisms. J. Prop. Power 19 (5), $722-734$.

[13] Garrido-Lopez, D. \& Sarkar, S. 2005 Effects of imperfect premixing coupled with hydrodynamic instability on flame propagation. Proc. Comb. Inst. 30, 621-627.

[14] Harrje, D. \& Reardon, F. 1972 Liquid propellant rocket combustion instability. NASA Rep. SP-194.

[15] Harten, A.V., Kapila, A.K. \& Matkowsky, B.J. 1984 Acoustic coupling of flames. SIAM J. App. Math. 44, 982-995.

[16] Jimenez, C., Cuenot, B., Poinsot, T. \& Howarth D. 2002 Numerical simulation and modelling for lean stratified propane-air flames. Combust. Flame 128, 1-21. 
[17] Jones, C.M., LEE, J.G. \& SantavicCA, D.A. 1999 Closed-loop active control of combustion instabilities using subharmonic secondary fuel injection. J. Propulsion Power 15(4), 584-590.

[18] Joulin, G. \& Cambray, P. 1992 On a tentative, approximate evolution equation for markedly wrinkled premixed flames. Comb. Sci. Technol. 81, 243-256.

[19] Langhorne, P.J. 1988 Reheat buzz: an acoustically coupled combustion instability. Part I. Experiment. J. Fluid Mech. 193, 417-443.

[20] Lee, J.G., Kim, K. \& Santavicca, D.A. 2000 Measurement of equivalence ratio fluctuation and its effect on heat release during unstable combustion. Proc. Comb. Inst. 28, 415-421.

[21] Lieuwen, T. 2003 Modeling premixed combustion-acoustic wave interactions: a review. J. Prop. Power 19(5), 765-781.

[22] Lieuwen, T., Torres, H., Johnson, C. \& Zinn, B.T. 2001 A mechanism of combustion instability in lean premixed gas turbine combustors. ASME J. Eng. Gas Turbine 123, 182-189.

[23] Lieuwen, T. \& Zinn, B.T. 1998 The role of equivalence ratio oscillations in driving combustion instabilities in low $N O_{x}$ gas turbines. Proc. Comb. Inst. 27, 1809-1816.

[24] Markstein, G.H. 1953 Instability phenomena in combustion waves. Proc. Comb. Inst. 4, 44-59.

[25] Markstein, G.H. \& Squire, W. 1955 On the stability of a plane flame front in oscillatory flow. J. American Acoustic Soc. 27(3), 416-424.

[26] Matalon, M. 2007 Intrinsic flame instability in premixed and nonpremixed combustion. Ann. Rev. Fluid Mech. 39, 163-191.

[27] Matalon, M. \& Matkowsky, B.J. 1982 Flames as gasdynamic discontinuities. J. Fluid Mech. 124, 239-259.

[28] Matalon, M. \& Metzener, P. 1997 The propagation of premixed flames in closed tubes. 336, 331-350.

[29] Matkowsky, B.J. \& Sivashinsky, G.I. 1979 An asymptotic derivation of two models in flame theory associated with the constant density approximation. SIAM J. Appl. Math. 37, 686-699.

[30] McIntosh, A.C. 1991 Pressure disturbances of different length scales interacting with conventional flames. Comb. Sci. Technol. 75, 287-309.

[31] McIntosh, A.C. 1993 The linearised response of the mass burning rate of a premixed flame to rapid pressure changes. Comb. Sci. Technol. 91, 3329-346.

[32] Mongia, B., Dibble, R. \& Lovett, J. 1998 Measurement of air-fuel fluctuations caused by combustor driven oscillations. ASME Paper, 98-GT-304.

[33] Mikolaitis, D. 1984 The unsteady propagation of premixed flames through nonhomogeneous mixtures and thermal gradient. Combust. Flame 57, 87-94. 
[34] Pelce, P. \& Clavin, P. 1982 Influence of hydrodynamics and diffusion upon the stability limits of laminar premixed flames. J. Fluid Mech. 124, 219-237.

[35] Pelce, P. \& Rochwerger, D. 1992 Vibratory instability of cellular flames propagating in tubes. J. Fluid Mech. 239, 293-307.

[36] Peter, N. 2000 Turbulent Combustion. Cambridge University Press.

[37] Peters, N. \& Ludford, G.S.S. 1984 The effect of pressure variations on premixed flames. Comb. Sci. Technol. 34, 331-344.

[38] Pitsch, H. 2005 A consistent level set formulation for large-eddy simulation of premixed turbulent combustion. Combust. Flame 133, 587-598.

[39] Poinsot, T.J., Trouve, A.C., Veynante, D.P., Candel, S.M. \& Esposito, E.J. 1987 Vortex-driven acoustically coupled combustion instabilities. J. Fluid Mech. 177, 265-292.

[40] Rahibe, M., Aubry, N. \& Sivashinsky, G.I. 1996 Stability of pole solutions for planar propagating flames. Phys. Rev. E 54, 4958-4972.

[41] Rastigejev, Y. \& Matalon, M. 2006 Nonlinear evolution of hydrodynamically unstable premixed flames. J. Fluid Mech. 554, 371-392.

[42] Richards, G.A. \& Janus, M. 1997 Characterization of oscillations during gas turbine combustion. ASME paper 97-GT-244.

[43] Richards, G.A. \& Janus, M. \& Robey, E.H. 1999 Control of flame oscillations with equivalence ratio modulation. J. Propulsion Power 15(2), 232-240.

[44] Schadow, K.C. \& Gutmark, E. 1992 Combustion instability related to vortex shedding in dump combustors and their passive control. Prog. Energy Combus. Sci. 18, 117-132.

[45] Schuller, T.D., Ducruix, S., Durox, D. \& Candel, S. 2002 Modeling tools for the prediction of premixed flame transfer functions. Proc. Comb. Inst. 29, 107-113.

[46] Schuller, T.D., Durox, D. \& Candel, S. 2003 A unified model for the prediction of flame transfer functions comparisons between conic and V-flame dynamics. Combust. Flame 134, 21-34.

[47] Searby, G. 1992 Acoustic instability in premixed flames. Comb. Sci. Technol. 81, 221-231.

[48] Searby, G. \& Clavin, P. 1986 Weakly turbulent, wrinkled flames in premixed gases. Comb. Sci. Technol. 46, 167-193.

[49] Searby, G. \& Rochwerger, D. 1991 A parametric acoustic instability in premixed flames. J. Fluid Mech. 231, 529-543.

[50] Sivasegaram, S., Tsai, R.F. \& Whitelaw, J.H. 1995 Control of combustion oscillations by forced oscillation of part of fuel supply. Comb. Sci Technol. 105, 6783. 
[51] Sivasegaram, S. \& Whitelaw, J. 1987 Oscillations in asixsymmeric dump combustors. Comb. Sci. Technol. 52, 413-426.

[52] Sivashinsky, G.I. 1977 Nonlinear analysis of hydrodynamic instability in laminar flames -I. Derivation of basic equations. Acta Astronautica 4, 1177-1206.

[53] Tachibana, S., Zimmer, L., Kurosawa, Y. \& Suzuki, K. 2007 Active control of combustion oscillations in a lean premixed combustor by secondary fuel injection coupling with chemiliminescence imaging technique. Proc. Comb. Inst. 31, 3225-3233.

[54] Vaynblat, D. \& Matalon, M. 1999 Stability of pole solutions for planar propagating flames: I. Exact eigenvalues and eigenfunctions. SIAM J. Appl. Math. 60, 679-702.

[55] Weigand, P., Meier, W., Duan, X. \& Aigner, M. 2007 Laser-based investigation of thermoacoustic instabilities in a lean premixed gas turbine. ASME J. Eng. Gas Turbine 129, 664-671.

[56] Wu, X. 2005 Asymptotic approach to combustion instability. Phil. Trans. R. Soc. Lond. 363, 1247-1259.

[57] Wu, X. \& LAw, C.K. 2009 Flame-acoustic resonance initiated by vortical disturbances. J. Fluid Mech. 637, 173-211.

[58] Wu, X \& Moin, P. 2008 Large-activation-enegry theory for premixed combustion under the influence of enthalpy fluctuations in the oncoming mixture. Part I: General formulation. Center Turbulence Research Ann. Rev. Res. Briefs, 403-419.

[59] Wu, X. Wang, M., Moin, P. \& Peters, N. 2003 Combustion instability due to the nonlinear interaction between sound and flame. J. Fluid Mech. 497, 23-53.

[60] Yu, K. H., Trouve, A. \& Daily, J.W. 1991 Low-frequency pressure oscillations in a model ramjet combustor. J. Fluid Mech. 232, 47-72.

[61] Zimmer, L. \& Tachibana, S. 2007 Laser induced plasma spectroscopy for local equivalence ratio measuremenrs in an oscillating combustion environment. Proc. Comb. Inst. 31, 737-745. 